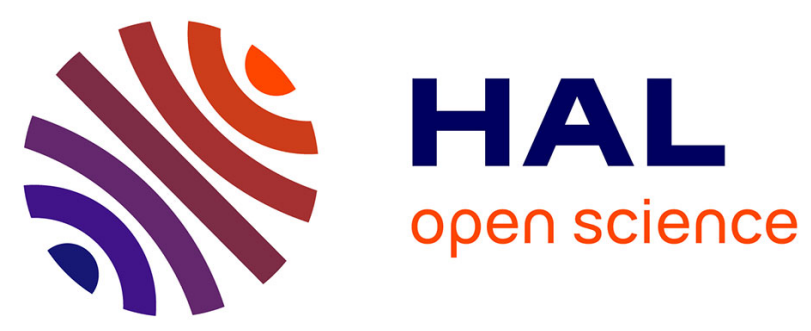

\title{
Interaction of two-dimensional spots with a heat releasing/absorbing shock wave: linear interaction approximation results
}

G. Farag, Pierre Boivin, P. Sagaut

\section{- To cite this version:}

G. Farag, Pierre Boivin, P. Sagaut. Interaction of two-dimensional spots with a heat releasing/absorbing shock wave: linear interaction approximation results. Journal of Fluid Mechanics, 2019, 871, pp.865-895. 10.1017/jfm.2019.324 . hal-02142649

\section{HAL Id: hal-02142649 \\ https://hal.science/hal-02142649}

Submitted on 28 May 2019

HAL is a multi-disciplinary open access archive for the deposit and dissemination of scientific research documents, whether they are published or not. The documents may come from teaching and research institutions in France or abroad, or from public or private research centers.
L'archive ouverte pluridisciplinaire HAL, est destinée au dépôt et à la diffusion de documents scientifiques de niveau recherche, publiés ou non, émanant des établissements d'enseignement et de recherche français ou étrangers, des laboratoires publics ou privés. 


\title{
Interaction of $2 \mathrm{D}$ spots with a heat releasing/absorbing shock wave: Linear Interaction Approximation results
}

\author{
G. Farag $^{1}$ P. Boivin ${ }^{1} \dagger$, and P. Sagaut ${ }^{1}$ \\ ${ }^{1}$ Aix Marseille Univ, CNRS, Centrale Marseille, M2P2 UMR 7340, Marseille, France
}

(Received xx; revised xx; accepted xx)

The canonical interaction between a two-dimensional weak Gaussian disturbance (entropy spot, density spot, weak vortex) with an exothermic/endothermic planar shock wave is studied via the Linear Interaction Approximation. To this end, a unified framework based on an extended Kovasznay decomposition that simultaneously accounts for non-acoustic density disturbances along with a poloidal-toroidal splitting of the vorticity mode and for heat-release is proposed. An extended version of Chu's definition for the energy of disturbances in compressible flows encompassing multi-component mixtures of gases is also proposed. This new definition precludes spurious non-normal phenomena when computing the total energy of extended Kovasznay modes. Detailed results are provided for three cases, along with fully general expressions for mixed solutions that combine incoming vortical, entropy and density disturbances.

\section{Introduction}

The propagation of a hydrodynamic shock wave across an heterogeneous medium is a very important topic in many fields of application, e.g. aerospace engineering, nuclear engineering but also astrophysics. Such an interaction is known to emit a complex field, which is a mixture of acoustic, entropy and vortical waves according to Kovasznay's decomposition (see Kovasznay (1953); Chu \& Kovásznay (1958); Sagaut \& Cambon (2018)). In the limit of small disturbances, the emitted field can be accurately predicted considering a linearized theory, namely the Linear Interaction Approximation (LIA), see Sagaut \& Cambon (2018) for an exhaustive discussion. This approximation is relevant in the wrinkled shock regime, in which the shock front corrugation by upstream disturbances is small enough to leave its topology unchanged, so that it can be decomposed as a linear sum of sinusoidal contributions. Several semi-empirical criteria of validity of LIA have been proposed on the ground of Direct Numerical Simulation results. In the case of a turbulent upstream flow, Lee et al. (1993) proposed

$$
M_{t}^{2}<0.1\left(M_{1}^{2}-1\right)
$$

where $M_{t}$ and $M_{1}$ are the upstream turbulent and mean Mach numbers, respectively. This criterion was later refined using DNS with higher resolution by Ryu \& Livescu (2014), yielding

$$
M_{t_{2}} \leqslant 0.1 M_{2}
$$

with $M_{t_{2}}$ and $M_{2}$ the downstream (LIA-predicted) turbulent Mach number and the

$\dagger$ Email address for correspondence: pierre.boivin@univ-amu.fr 
downstream mean flow based Mach number, respectively. In the laminar case of the interaction between an entropy spot and a normal shock, (Fabre et al. 2001) reported an excellent agreement within $1 \%$ error up to $M_{1}=4$ for disturbances with relative amplitude lesser or equal to 0.01 .

This theory was pioneered in the 1950s by Ribner (1954a, $\underline{b}, 1959)$; Moore (1953) and is still under development. The most complete formulation of the normal mode analysis for canonical interaction was given by Fabre et al. (2001), which was further extended to the case of the non-reacting binary mixture of perfect gas (Griffond 2005; Griffond et al. 2010) and to rarefaction waves (Griffond \& Soulard 2012). Following this approach, wave vectors of emitted waves are obtained analytically thanks to the dispersion relation stemming from the linearized Euler equations, while wave amplitudes are solution of a linear system. A deeper physical insight is obtained by grouping upstream disturbances according to the Kovasznay normal-mode decomposition of small compressible fluctuations into acoustic, vorticity and entropy mode. This decomposition has been extended by splitting the vorticity mode as the sum of a poloidal and a toroidal components (Griffond \& Soulard 2012), and also considering a binary mixture of perfect gas (Griffond 2005, 2006). Several cases have been succesfully investigated using LIA, among which the case of an upstream entropy spot (Fabre et al. 2001), upstream vortical isotropic turbulent field (Lee et al. 1993, 1997; Quadros et al. 2016), upstream isotropic acoustic turbulent field (Mahesh et al. 1995), upstream isotropic mixed vortical-entropy turbulent field (Mahesh et al. $\overline{1997)}$.

An alternative complete analytical treatment of the linearized problem based on the Laplace transform has been developed by Wouchuk, Huete and coworkers in a series of papers (e.g. Wouchuk et al. 2009; de Lira 2010; Huete et al. 2012a,ba, 2013). Here, a telegraphist equation is obtained for each type of incident wave whose analytical solution gives the amplitude of emitted disturbances. This approach has not been explicitly recast into Kovasznay framework up to now, but acoustic and vortical upstream fluctuations have been considered in a series of papers, along with density fluctuations. The analysis has been recently extended to the case of thin detonation waves (Huete et al. 2013, 2014), which are described as shock wave associated to a heat release phenomenon. That approach has also been applied to many cases, e.g. incident isotropic adiabatic turbulence (Wouchuk et al. 2009), pure incident acoustic turbulence (Huete et al. 2012b), pure incident isotropic density fluctuations including the re-shock problem (Huete et al. $2012 \underline{\mathrm{a}}$ ).

Selected studies carried out within these two general frameworks are listed in Table 1 in an attempted summary, sorting the studies referred to in the two previous paragraphs according to the perturbation modes considered, the possibility to account for heat releasing/absorbing shock, as well as the upstream perturbations and the approach followed. It is worth noting that in the case of an upstream turbulent field, LIA can be rewritten in terms of turbulent fluxes, leading to a linear problem for the jump of these quantities across the shock. These relations can be used to derive RANS models well suited for the simulation of the shock-turbulence interaction (Sinha et al. 2003; Griffond et al. 2010; Soulard et al. 2012; Sinha 2012; Quadros et al. 2016).

The goal of the present paper is three-fold. First, it aims at providing a complete, unified formulation of the normal-mode-based LIA approach that encompasses all previous developments, namely binary mixture of perfect gas interacting with a nonadiabatic shock wave considering the poloidal/toroidal splitting of vorticity. The various extensions mentioned above have not been gathered into a single unified framework up to now. In particular, accounting for the non-adiabatic character of a shock wave simultaneously with these extensions has not been done up to now, although it was 


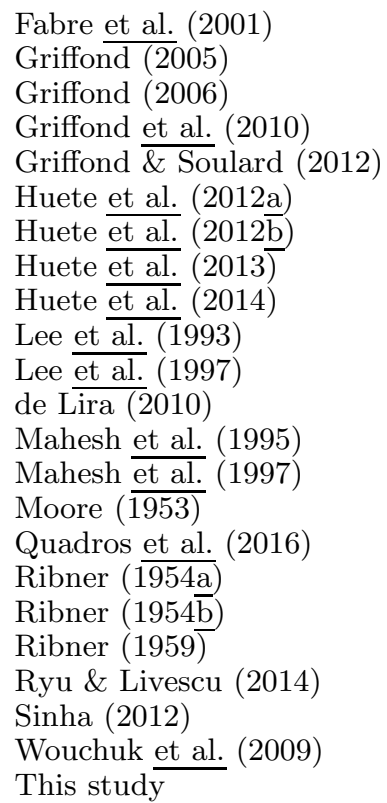

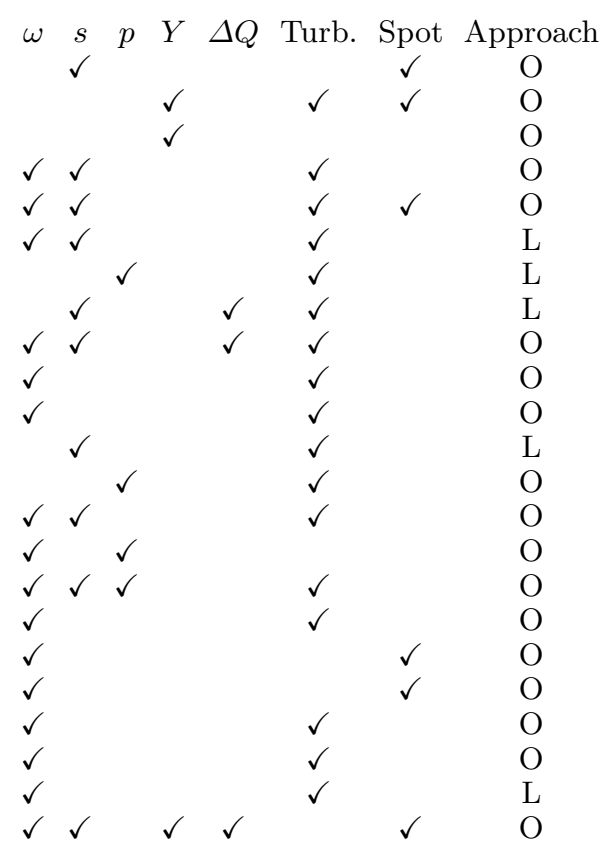

Table 1: Summary of the LIA literature. $\omega, s, p$ and $Y$ indicate the considered incident Kovásznay modes, and $\Delta Q$ the presence of a heat releasing and/or absorbing shock. Turb (turbulent) and Spot refer to the nature of the upstream field. The approach followed is also indicated as $\mathrm{L} / \mathrm{O}$, referring respectively studies articles based/not based on the Laplace transform.

carried out in the case of density fluctuations through detonations (Huete et al. 2013, 2014). Heat-release/absorption will be described as a punctual source/sink at the shock, to encompass thin reactive shock waves, shock-induced condensation or radiative loss (see e.g. Zel'Dovich \& Raizer 2012). In this general formulation, all types of upstream disturbances will be considered within an extended Kovasznay decomposition framework.

The second goal of the paper is to extend Chu's definition for disturbance energy (Chu 1965) to a multi-component fluid: a physically relevant and mathematically consistent definition well-suited for small perturbations definition of the disturbance energy is of primary importance to analyze the effect of the interaction with the shock wave, and is therefore a prerequisite to the next paper's goal.

The last goal of the present paper is to analyze the interaction of a Gaussian perturbation spot with a shock wave in the presence of phenomena mentioned above. Three different cases are investigated: a density spot, an entropy spot and a vorticity spot (i.e. a weak vortex). It is worth noting that the case of upstream density heterogeneities has been considered in the case of non-reactive shock waves and thin strong detonations by Huete et al. (2013). Such a simple configuration can be considered as an idealized model of the interaction of a shock wave with a two-phase heterogeneity (bubble, droplet) with small density ratio. To the knowledge of the authors, such general cases have never been considered in the open literature up to now. Using the three elementary cases considered in the present papers, an infinite number of cases can be derived by linear combination 
of the LIA results. As an example, the interaction between a shock wave and a cold weak vortex is obtained in a straightforward way by linear combination of the solutions related to the isentropic vortex case and a cold entropy spot. Multiple spot solutions can also be found in the same way, introducing a space-time shift in the solution associated to each spot. The optimal combination of these elementary spots to minimize the radiated noise is investigated in the present paper.

The paper is organized as follows. The basic physical model and associated governing equations are displayed in Section 2. The decomposition of both upstream and downstream fields according to the present extended Kovasznay modal decomposition is then presented in Section 3. The extended definition of disturbance energy and its relation to the energy of Kovasznay modes are discussed in Section 4. Then the proposed general formulation of the normal-mode-based LIA approach is discussed in Section 5. The application to the interaction of a heat releasing/absorbing shock wave with a variety of Gaussian spots (for density, entropy and vorticity fluctuations) is then addressed in Section 6, with most of the technical details regarding the treatment of 2D Gaussian spots given in Appendix. Conclusions are drawn in Section 7.

\section{Physical model}

The physical model addressed in the present paper is related to the case of $2 \mathrm{D}$ canonical shock/disturbance interaction in a binary mixture of perfect gas, in the presence of heat release/absorption on the shock wave. Viscous effects are neglected. Upstream and downstream of the normal shock, the flow is governed by the Euler equations:

$$
\begin{cases}\frac{\partial \rho}{\partial t}+\operatorname{div}(\rho \mathbf{u}) & =0 \\ \frac{\partial \rho \mathbf{u}}{\partial t}+\operatorname{div}(\rho \mathbf{u} \otimes \mathbf{u}+p \underline{I}) & =0 \\ \frac{\partial \rho E}{\partial t}+\operatorname{div}((\rho E+p) \mathbf{u}) & =0 \\ \frac{\partial \rho Y}{\partial t}+\operatorname{div}(\rho Y \mathbf{u}) & =0\end{cases}
$$

where $p, \rho, \mathbf{u}$ and $E$ denote the mixture pressure, density, velocity, and total energy; and $Y$ is the mass fraction of the first component in the binary mixture (see e.g. Williams 1985).

The mixture equation of state for the binary mixture reads

$$
p=\rho \frac{R}{W} T,
$$

where $R$ and $W$ denote the perfect gas constant and the molar weight of the mixture, respectively. The classical relations for ideal gas mixtures yields the following relations between the component properties and the mixture properties:

$$
\begin{gathered}
1 / W=Y / W_{a}+(1-Y) / W_{b}, \\
c_{v}=Y c_{v, a}+(1-Y) c_{v, b}, \quad \text { and } \quad c_{p}=Y c_{p, a}+(1-Y) c_{p, b}, \\
\gamma_{a}=\frac{c_{p, a}}{c_{v, a}}, \quad \gamma_{b}=\frac{c_{p, b}}{c_{v, b}}, \quad \gamma=\frac{c_{p}}{c_{v}}, \\
A_{t}^{r}=\frac{r_{a}-r_{b}}{\bar{r}}, \quad \text { and } \quad A_{t}^{c_{v}}=\frac{c_{v a}-c_{v b}}{\bar{c}_{v}} .
\end{gathered}
$$


$W, c_{v}, c_{p}$ and $\gamma$ denote respectively the mixture molecular weight, mass heat capacity at constant volume and constant pressure, the heat capacity ratio, as well as two Atwood numbers, to be used hereafter. Subscripts $a$ and $b$ denote the corresponding component thermodynamic properties in the binary mixture, one being inert and one possibly reactive. Note however that they do not intervene in the following, where indices exclusively serve as to identify the upstream and downstream states.

Considering the case of an 1D flow along the $x$ axis and a normal shock wave and denoting $\left(u_{x}, u_{r}, u_{\phi}\right)$ the component of velocity in cylindrical coordinates (in the discontinuity reference frame, the $x$ axis being taken normal to the planar shock wave), the upstream and downstream mean quantities (resp. subscripts 1,2) relate through the Hugoniot jump conditions for mass, momentum and energy:

$$
\begin{aligned}
\rho_{1} u_{x 1} & =\rho_{2} u_{x 2}, \\
p_{1}+\rho_{1} u_{x 1}^{2} & =p_{2}+\rho_{2} u_{x 2}^{2}, \\
h_{1}+\frac{u_{x 1}^{2}}{2} & =h_{2}+\frac{u_{x 2}^{2}}{2},
\end{aligned}
$$

with $u_{r}, u_{\phi}$ and $Y_{a}$ being conserved through the shock:

$$
u_{r 1}=u_{r 1}, \quad u_{\phi 1}=u_{\phi 2}, \quad Y_{a, 1}=Y_{a, 2} .
$$

The enthalpy $h$ jump condition may be reformulated as

$$
c_{p} T_{1}+\frac{u_{x 1}^{2}}{2}=c_{p} T_{2}+\frac{u_{x 2}^{2}}{2}-\Delta Q,
$$

where $\Delta Q$ accounts for heat release/heat absorption at the shock wave.

$\Delta Q>0$ was considered by Huete et al. (2013) to model thin detonations, while $\Delta Q<0$ should be used to account for physical mechanisms restricted to a thin region downstream the shock front that act as an energy sink, e.g. radiative losses or condensation (Zel'Dovich \& Raizer 2012).

Note that, while $\Delta Q$ is here formulated as an independent parameter, a classical assumption for strong detonations (see, e.g. Williams 1985), the heat absorption typically depends on the shock strength for endothermic processes (which typically ends when saturation is reached), as is the case in ionizing, nuclear dissociating shocks as those occurring in core collapsing supernovae (Huete et al. 2018; Abdikamalov et al. 2018; Huete \& Abdikamalov 2019), shock-induced condensation in vapor-liquid two-phase flow (Zhao et al. 2008) or cooling induced by radiative loss (Narita 1973).

Introducing the sound speeds on either side of the shock $c_{1}, c_{2}$ in the jump conditions lead to the following relation between the upstream and downstream Mach Numbers, respectively $M_{1}$ and $M_{2}$ :

$$
M_{2}^{2}=\frac{1+\gamma M_{1}^{2}-\left(M_{1}^{2}-1\right) \sqrt{1-\beta}}{1+\gamma M_{1}^{2}+\gamma\left(M_{1}^{2}-1\right) \sqrt{1-\beta}}, \quad \beta=\frac{2\left(\gamma^{2}-1\right) M_{1}^{2} q}{\left(1-M_{1}^{2}\right)^{2}},
$$

where the normalized heat coefficient has been introduced

$$
q=\frac{\Delta Q}{c_{1}^{2}}
$$

The compression factor $m=\rho_{2} / \rho_{1}=u_{1} / u_{2}$ is obtained through

$$
\frac{1}{m}=\frac{1+\gamma M_{1}^{2}}{(\gamma+1) M_{1}^{2}}+\frac{1-M_{1}^{2}}{(\gamma+1) M_{1}^{2}} \sqrt{1-\beta}
$$

Note that $c_{p}$ and $\gamma$, appearing in the above relations are identical on both side of the 

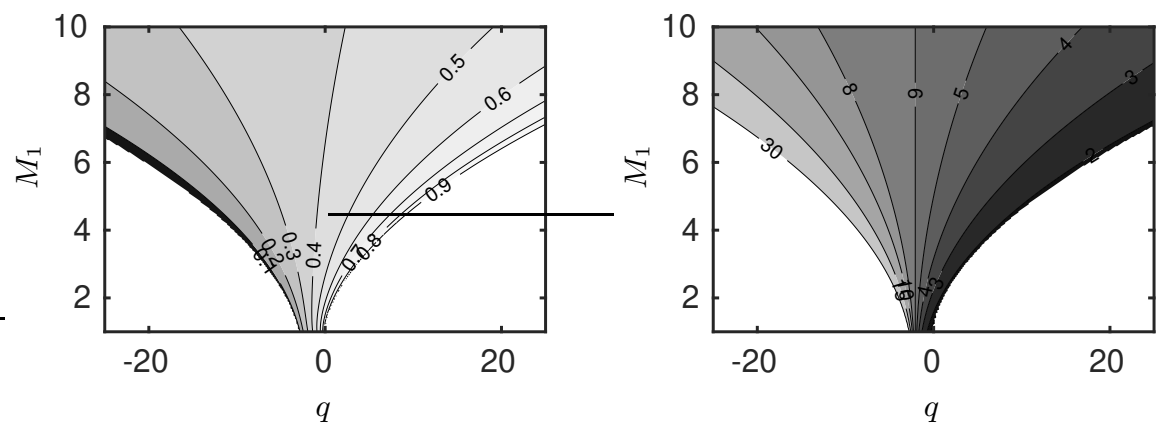

Figure 1: Downstream Mach number $M_{2}$ (left) and compression factor $m$ (right) as functions of the upstream Mach number $M_{1}$ and the heat source/sink parameter $q$ according to Eqs.(2.6) to (2.10).

shock thanks to the continuity of mass fraction $Y$, thereby considerably reducing the equations. A detailed account on the validity of this assumption has been provided by Griffond (2005): the analysis is valid for small concentration fluctuations within binary mixtures with very different thermodynamic properties, or large concentration fluctuations within gases of similar thermodynamic properties. This translates, in practice, to the assumption holding when the reactive component mixture is sufficiently dilute in the inert one, as is often the case in air. When the assumption does not hold, the present study still present valuable benchmarks for numerical codes, in which thermodynamic properties may be artificially set to constants.

All other classical relations for $T_{2} / T_{1}, p_{2} / p_{1}, \ldots$ are formally identical to those of the classical normal shock case, $M_{2}$ and $m$ being now given by the above formula.

The consistency constraint which ensures that both $m$ and $M_{2}$ remain positive is

$$
q_{\min }<q<q_{\max }
$$

where

$$
q_{\min }=\frac{1}{1-\gamma}-\frac{M_{1}^{2}}{2}, \quad q_{\max }=\frac{\left(1-M_{1}^{2}\right)^{2}}{2\left(\gamma^{2}-1\right) M_{1}^{2}} .
$$

The consistent domain for heat-source/sink as a function of the upstream Mach number $M_{1}$ is illustrated in Fig. 1. Superimposed are contours of the downstream Mach number $M_{2}$, as provided by (2.6). One recovers the physical behavior that the downstream flow is accelerated in the case $q>0$ compared to the neutral shock case $q=0$, while it is decelerated in the opposite case $q<0$, due to the balance between kinetic energy and internal energy. In the asymptotic limit $q=q_{\max }$, the system satisfies the so-called Chapman-Jouguet condition $M_{2}=1$ (see, e.g. Zeldovich 1950). The other limit, $q=q_{\text {min }}$ corresponds to an infinite mass compression ratio, impossible to sustain in practice. For this reason, the endothermic cases presented in Section 6 are presented for $q=$ $q_{\mathrm{min}} / 2$, translating to at most half the upstream kinetic energy being absorbed, leading to reasonable compression ratio and downstream Mach numbers (resp. $m=6.5$ and $\left.M_{2}=0.33\right)$. 


\section{The Kovasznay modal decomposition for disturbances in a binary mixture of ideal gas}

The Linear Interaction Approximation relies on a small disturbance hypothesis and the use of linearized equations to described fluctuation propagation on either side of the shock.

For each quantity (e.g. $u)$, let us identify the fluctuation part $\left(u^{\prime}\right)$ and the mean $(\bar{u})$ as

$$
u=\bar{u}+u^{\prime}, \quad p=\bar{p}+p^{\prime}, \quad \ldots
$$

and assume the fluctuation part is small $\left(u^{\prime} / \bar{u} \ll 1\right)$, a classical assumption provided:

- Linearization of $Y$, for which $\bar{Y}=0$ is acceptable, is valid (Griffond 2005). This is in practice related to the continuity of $c_{p}$ and $\gamma$ discussed after Eq. (2.8).

- Similarly, the linearization for the normal shock velocity is questionable in the limit $\bar{u} \rightarrow 0$, attainable when $q \rightarrow q_{\text {min }}$. To avoid this, the present study should not be carried out for $M_{2}<0.25$, or, alternatively, $q<q_{\min } / 2$.

In the reference frame tied to the planar shock front the $2 \mathrm{D}$ perturbation field then satisfies

$$
\begin{cases}\frac{\partial \rho^{\prime}}{\partial t}+\bar{u} \frac{\partial \rho^{\prime}}{\partial x}+\bar{\rho} \frac{\partial u_{j}^{\prime}}{\partial x_{j}} & =0 \\ \frac{\partial u_{i}^{\prime}}{\partial t}+\bar{u} \frac{\partial u_{i}^{\prime}}{\partial x}+\frac{1}{\bar{\rho}} \frac{\partial p^{\prime}}{\partial x_{i}} & =0 \\ \frac{\partial Y^{\prime}}{\partial t}+\bar{u} \frac{\partial Y^{\prime}}{\partial x} & =0 \\ \frac{\partial p^{\prime}}{\partial t}+\bar{u} \frac{\partial p^{\prime}}{\partial x}+\gamma \bar{p} \frac{\partial u_{j}^{\prime}}{\partial x_{j}} & =0\end{cases}
$$

which can be recast as a system of evolution equations for Kovasznay's physical modes:

$$
\left\{\begin{array}{c}
\frac{\partial s^{\prime}}{\partial t}+\bar{u} \frac{\partial s^{\prime}}{\partial x}=0 \\
\frac{\partial \omega_{\|}^{\prime}}{\partial t}+\bar{u} \frac{\partial \omega_{\|}^{\prime}}{\partial x}=0, \\
\frac{\partial \omega_{\perp}^{\prime}}{\partial t}+\bar{u} \frac{\partial \omega_{\perp}^{\prime}}{\partial x}=0, \\
\left(\frac{\partial}{\partial t}+\bar{u} \frac{\partial}{\partial x}\right)^{2} p^{\prime}=c^{2} \nabla^{2} p^{\prime} \\
\frac{\partial Y^{\prime}}{\partial t}+\bar{u} \frac{\partial Y^{\prime}}{\partial x}=0,
\end{array}\right.
$$

where $\omega^{\prime}=\nabla \times \mathbf{u}^{\prime}$ denotes the fluctuating vorticity, and $\omega_{\perp}^{\prime}=\left(\omega^{\prime} \cdot \mathbf{n}\right) \mathbf{n}$ and $\omega_{\|}^{\prime}=\omega^{\prime}-\omega_{\perp}^{\prime}$ are the shock-normal and the shock-parallel components of vorticity, respectively, with $\mathbf{n}$ the unit normal vector of the planar shock wave. The shock-normal and the shocktangential components correspond to the toroidal and poloidal components of the velocity field in the reference frame tied to the planar shock front, respectively.

One recognizes the entropy mode, the toroidal and poloidal vorticity modes, the fast and slow acoustic modes and the concentration mode. It is worth noting that Kovasznay's modes correspond to the eigenmodes of the linearized propagation operator, which are orthonormal according to the inner product associated to Chu's definition of compressible disturbance energy. 


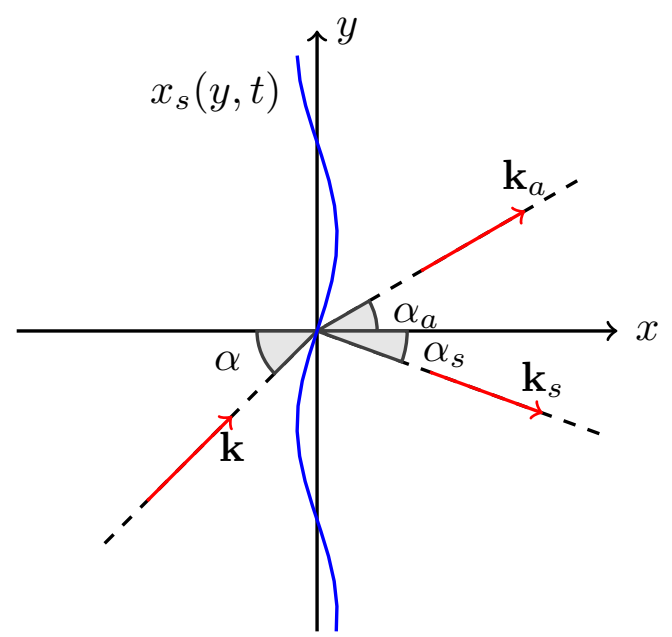

Figure 2: Sketch of the configuration. The corrugated shock mean front position is at $x=0$. The incident perturbation has wave vector $\mathbf{k}$, at angle $\alpha$ with respect to the shock normal. Emitted waves may be acoustic waves, with wave vector $\mathbf{k}_{a}$, or non-acoustic ones, with wave vector $\mathbf{k}_{s}$.

Let us now introduce propagating plane wave disturbances of the general form

$$
\phi^{\prime}=A_{i}(\mathbf{k}) \exp [\mathrm{i}(\mathbf{k} \cdot \mathbf{x}-\Omega t)]
$$

Here, $A_{i}(\mathbf{k})$ denotes the amplitude of upstream Kovasznay mode of type $i$, with $i=$ $s, a, Y, v, t$ for entropy, acoustic, concentration and poloidal/toroidal vorticity mode, respectively. $\mathbf{k}$ is the perturbation wave vector, associated with pulsation $\Omega=\bar{u}_{1} k \cos \alpha$, where $\alpha$ is the angle of the incident perturbation with respect to the shock, as illustrated in Fig. 2.

The upstream fluctuating field can then be decomposed as follows

$$
\left[\begin{array}{c}
\tau_{1}^{\prime} / \bar{\tau}_{1} \\
u_{x 1}^{\prime} / \bar{u}_{1} \\
u_{r 1}^{\prime} / \bar{u}_{1} \\
u_{\phi 1}^{\prime} / \bar{u}_{1} \\
p_{1}^{\prime} / \gamma \bar{p}_{1} \\
Y_{1}^{\prime} \\
T_{1}^{\prime} / \bar{T}_{1} \\
s_{1}^{\prime} / C_{p 1}
\end{array}\right]=A_{i}(\mathbf{k}) e^{\mathrm{i}(\mathbf{k} \cdot \mathbf{x}-\Omega t)}\left[\begin{array}{c}
\delta_{i s}-\delta_{i a}+\delta_{i Y} A_{t}^{r} \\
\delta_{i v} \sin \alpha+\delta_{i a} \frac{\cos \alpha}{M_{1}} \\
-\delta_{i v} \cos \alpha+\delta_{i a} \frac{\sin \alpha}{M_{1}} \\
\delta_{i t} \\
\delta_{i a} \\
\delta_{i Y} \\
\delta_{i s}+(\gamma-1) \delta_{i a} \\
\delta_{i s}
\end{array}\right],
$$

where $\delta_{i j}$ is the Kronecker symbol, and $\tau=1 / \rho$ is the specific volume.

Now introducing the transfer function $Z_{i j}$ between upstream Kovasznay mode of type $i$ and downstream Kovasznay mode of type $j$, the emitted fluctuating field downstream the shock is given by: 


$$
\begin{gathered}
{\left[\begin{array}{c}
\tau_{2}^{\prime} / \bar{\tau}_{2} \\
u_{x 2}^{\prime} / \bar{u}_{2} \\
u_{r 2}^{\prime} / \bar{u}_{2} \\
u_{\phi 2}^{\prime} / \bar{u}_{2} \\
p_{2}^{\prime} / \gamma \bar{p}_{2} \\
Y_{2}^{\prime} \\
T_{2}^{\prime} / \bar{T}_{2} \\
s_{2}^{\prime} / C_{p 2}
\end{array}\right]=A_{i}(\mathbf{k}) e^{-k_{a} \eta x} e^{\mathrm{i}\left(\mathbf{k}_{a} \cdot \mathbf{x}-\Omega t\right)}\left[\begin{array}{c}
-Z_{i a} \\
Z_{i a}\left(\cos \alpha_{a}+\mathrm{i} \eta\right) /\left(M_{2} \zeta\right) \\
Z_{i a} \sin \alpha_{a} /\left(M_{2} \zeta\right) \\
0 \\
Z_{i a} \\
0 \\
(\gamma-1) Z_{i a} \\
0 \\
+A_{i}(\mathbf{k}) e^{\mathrm{i}\left(\mathbf{k}_{s} \cdot \mathbf{x}-\Omega t\right)}\left[\begin{array}{c}
Z_{i s}+Z_{i Y} A_{t}^{r} \\
Z_{i v} \sin \alpha_{s} \\
-Z_{i v} \cos \alpha_{s} \\
Z_{i t} \\
0 \\
Z_{i Y} \\
Z_{i s} \\
Z_{i s}
\end{array}\right],
\end{array}\right.}
\end{gathered}
$$

where

$$
\zeta=\sqrt{1-\eta^{2}+2 \mathrm{i} \eta \cos \alpha_{a}}
$$

Acoustic and non-acoustic emitted fluctuations are separated into two contributions in Eq. (3.5), as they correspond to different wave vectors, resp. $\mathbf{k}_{a}$ (possibly associated to attenuation $\eta$ ) and $\mathbf{k}_{s}$. These wave vectors are explicited hereafter. The transfer function $Z_{i j}$ coefficients are explicitly given in Section 5 .

\section{Emitted acoustic and non-acoustic wave vectors}

Evaluation of the wave vectors $\mathbf{k}_{a}, \mathbf{k}_{s}$ and the associated angles $\alpha_{a}, \alpha_{s}$ and attenuation $\eta$ is classical (see Fabre et al. 2001; Sagaut \& Cambon 2018), but is nonetheless recalled for the sake of completness.

The effect of $\alpha$ being different whether the incident perturbation is acoustic $(i=a)$ or non-acoustic $(i \neq a)$, it is convenient to introduce the modified incident angle $\beta$ as

$$
\beta=\delta_{i a} \alpha^{\prime}+\left(1-\delta_{i a}\right) \alpha
$$

where $\alpha^{\prime}$ is defined as

$$
\cot \alpha^{\prime}=\cot \alpha+\frac{1}{M_{1} \sin \alpha} .
$$

Wave vectors and angles are then related through the relation:

$$
\frac{k_{a, s}}{k}=\frac{\sin \beta}{\sin \alpha_{a, s}},
$$

valid for both acoustic and non-acoustic emitted waves.

The emitted non-acoustic wave vector angle simply reads

$$
\cot \alpha_{s}=m \cot \beta \text {, }
$$

where $m$ is the compression factor (2.8).

Obtaining the emitted acoustic wave vector $\mathbf{k}_{a}$ and associated attenuation $\eta$ is not as straightforward. If the incident perturbation is non-acoustic $(i \neq a)$, a singularity appears 


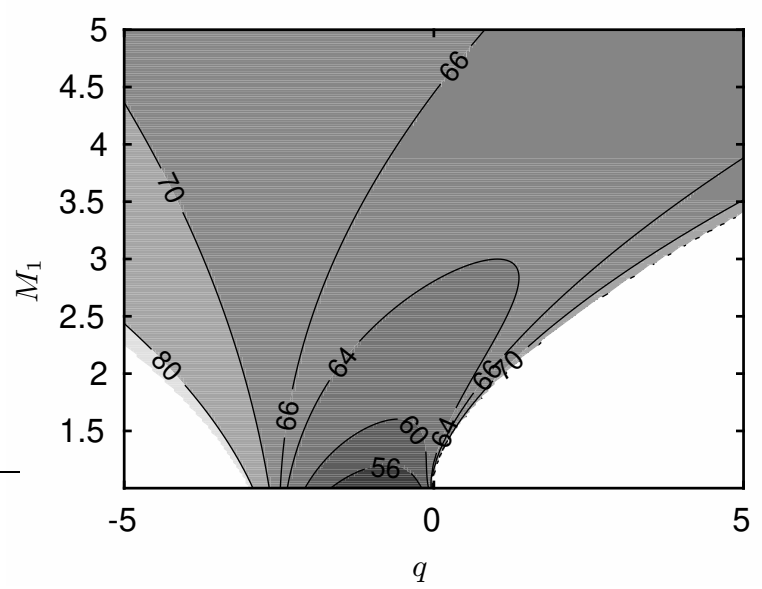

Figure 3: Isovalues of the critical angle $\alpha_{c}$ as a function of the upstream Mach number $M_{1}$ and the heat source term $q$. White areas correspond to unphysical configurations that violate the realizability constraint on the downstream flow.

for $\alpha=\beta=\alpha_{c}$, where

$$
\cot \alpha_{c}=\frac{\sqrt{1-M_{2}^{2}}}{m M_{2}}
$$

for which the emitted acoustic wave vector corresponds to the critical emission angle

$$
\cos \alpha_{a}^{c}=-M_{2} \text {. }
$$

If $\alpha<\alpha_{c}$, the emitted wave vector angle reads

$$
\frac{\cot \alpha_{a}}{\cot \alpha_{a}^{c}}=\frac{\cot \beta}{\cot \alpha_{c}}-\frac{1}{M_{2}} \sqrt{\left(\frac{\cot \beta}{\cot \alpha_{c}}\right)^{2}-1} \text { and } \eta=0,
$$

else if $\alpha>\alpha_{c}$ :

$$
\frac{\cot \alpha_{a}}{\cot \alpha_{a}^{c}}=\frac{\cot \beta}{\cot \alpha_{c}} \text { and } \eta=\frac{\left|\cot \alpha_{a}^{c} \sin \alpha_{a}\right|}{M_{2}}-\frac{1}{M_{2}} \sqrt{1-\left(\frac{\cot \beta}{\cot \alpha_{c}}\right)^{2}},
$$

In the particular case where the incident perturbation is acoustic $(i=a), \beta=\alpha^{\prime}$, and two critical values for the incident angle $\alpha$ are found:

$$
\alpha_{c}^{ \pm}+\frac{1}{M_{1} \sin \alpha_{c}^{ \pm}}=\mp \frac{\sqrt{1-M_{2}^{2}}}{m M_{2}},
$$

corresponding to fast and slow propagation regimes, separated by incident angle $\alpha_{M}$ such as

$$
\cos \alpha_{M}=-\frac{1}{M_{1}}
$$

For acoustic incident perturbations $\left(\beta=\alpha^{\prime}\right)$ Eqs. (3.13) and (3.14) remain valid, now defining four regimes: a propagating and non-propagating regime for each of the fast and slow modes.

The global procedure for the determination of emitted wave vectors as well as associated attenuation is summarized in Table 2 and the resulting dependence on the incident 


$$
\begin{aligned}
& \text { non-acoustic perturbation } \begin{cases}\forall \alpha, & \alpha_{s} \text { from Eq. (3.10) with } \beta=\alpha \\
\alpha<\alpha_{c}, & \left(\alpha_{a}, \eta\right) \text { from Eq. (3.13) with } \beta=\alpha \\
\alpha>\alpha_{c}, \quad\left(\alpha_{a}, \eta\right) & \text { from Eq. (3.14) with } \beta=\alpha\end{cases} \\
& \text { acoustic perturbation } \begin{cases}\forall \alpha, & \alpha_{s} \text { from Eq. (3.10) with } \beta=\alpha^{\prime} \\
0<\alpha<\alpha_{c}^{-}, & \left(\alpha_{a}, \eta\right) \text { from (3.13) with } \beta=\alpha^{\prime} \\
\alpha_{c}^{-}<\alpha<\alpha_{M}, & \left(\alpha_{a}, \eta\right) \text { from (3.14) with } \beta=\alpha^{\prime} \\
\alpha_{M}<\alpha<\alpha_{c}^{+}, & \left(\alpha_{a}, \eta\right) \text { from (3.14) with } \beta=\alpha^{\prime} \\
\alpha_{c}^{+}<\alpha<\pi, & \left(\alpha_{a}, \eta\right) \text { from (3.13) with } \beta=\alpha^{\prime}\end{cases}
\end{aligned}
$$

Table 2: Computation of the emitted acoustic and non acoustic wave vectors through the corresponding angles $\alpha_{a}$ and $\alpha_{s}$, for non-acoustic and acoustic incident perturbation. Also included is the determination of attenuation $\eta$ for emitted acoustic waves.
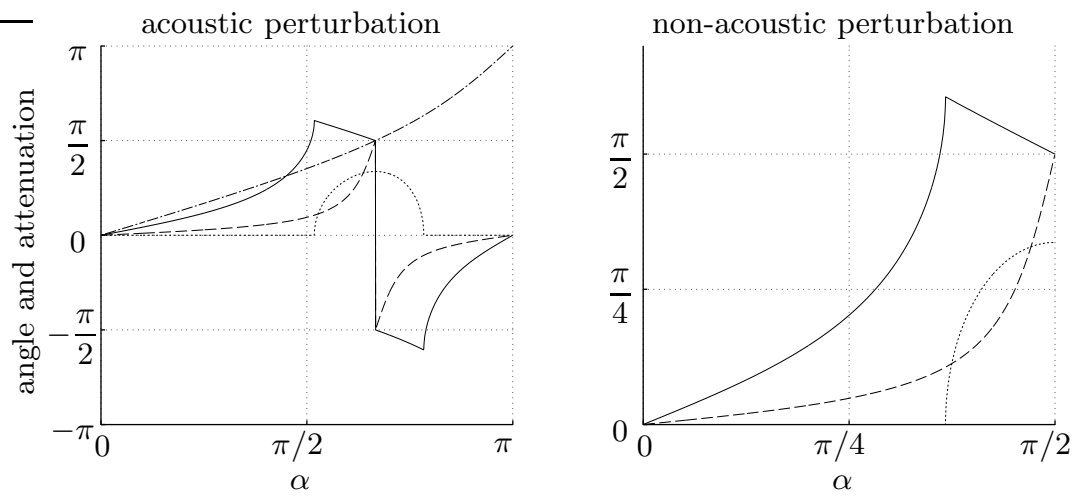

Figure 4: Emission angles and attenuation factor $\eta$, obtained following the procedure summarized in Tab. 2, for $\gamma=1.4, M_{1}=2$ and $q=-2.25$. Plain line: $\alpha_{a}$, dashed-line: $\alpha_{s}$, dotted-line: attenuation $\eta$. In the left plot, the additional dot-dashed line represents the non-linear dependence of $\beta$ as a function of $\alpha$ (3.7) in the case of an acoustic incident perturbation.

angle is illustrated in Fig. 4.

\section{Extension of Chu's definition for disturbance energy to multicomponent gas}

An important issue is the derivation of a physically relevant and mathematically consistent definition of the energy of the disturbances in compressible flows. Chu's definition (Chu 1965) for the disturbance energy around a base flow has the advantage to define an inner product, with respect to which the linearized Euler equations about a uniform base flow are self-adjoint, and Kovasznay modes correspond to orthogonal eigenmodes of the linearized operator. The orthogonality of eigenmodes prevents spurious non-normality-induced phenomenon in the computation of the energy of the fluctuating field (George \& Sujith 2011; Sagaut \& Cambon 2018) As a matter of fact, the use of a non-normal basis may lead to unphysical growth of the energy of the system because of the contributions of non-zero cross-products of basis vectors. Therefore, one can split 
the total energy as the sum $E_{t o t}=\sum_{i} E_{i}$, with $i=v, a, s$ for the vorticity mode, the acoustic mode and the entropy mode, respectively.

Since the present work deals with multi-component gas, the original Chu's definition is extended in the present section. A first step consists of finding an expression of the linearized Euler equations that will lead to orthogonal eigenvectors. This is the case when the matrix associated to the linearized problem is symmetric. To this end, an adequate choice of physical unknowns must be done. Noticing that the set $(\rho, u, v, T, Y)$ leads to a non-symmetric matrix and non-orthogonal eigenvectors, we choose here to write the linearized problem using $\left(\rho_{a}, \rho_{b}, u, v, T\right)$ :

$$
\left\{\begin{array}{l}
\frac{\partial \rho_{a}^{\prime}}{\partial t}+\bar{u} \frac{\partial \rho_{a}^{\prime}}{\partial x}+\bar{\rho}_{a} \frac{\partial u_{j}^{\prime}}{\partial x_{j}}=0 \\
\frac{\partial \rho_{b}^{\prime}}{\partial t}+\bar{u} \frac{\partial \rho_{b}^{\prime}}{\partial x}+\bar{\rho}_{b} \frac{\partial u_{j}^{\prime}}{\partial x_{j}}=0 \\
\frac{\partial u_{i}^{\prime}}{\partial t}+\bar{u} \frac{\partial u_{i}^{\prime}}{\partial x}+\frac{1}{\bar{\rho}} \frac{\partial p^{\prime}}{\partial x_{i}}=0 \\
\frac{\partial T^{\prime}}{\partial t}+\bar{u} \frac{\partial T^{\prime}}{\partial x}+\frac{\bar{p}}{\bar{\rho} C_{v}} \frac{\partial u_{j}^{\prime}}{\partial x_{j}}=0 \\
\frac{p^{\prime}}{\bar{p}}-\frac{T^{\prime}}{\bar{T}}-\left[1+A_{t}^{r}\left(1-Y_{0}\right)\right] \frac{\rho_{a}^{\prime}}{\rho_{0}}-\left(1-A_{t}^{r} Y_{0}\right) \frac{\rho_{b}^{\prime}}{\rho_{0}}=0
\end{array}\right.
$$

where the last line is related to the linearized equation of state, with

$$
\begin{gathered}
\rho_{a}=\rho Y \quad \rho_{b}=\rho(1-Y), \\
\frac{\rho_{a}^{\prime}}{\rho_{0}}=\frac{\rho^{\prime}}{\rho_{0}} Y_{0}+Y^{\prime}, \\
\frac{\rho_{b}^{\prime}}{\rho_{0}}=\frac{\rho^{\prime}}{\rho_{0}}\left(1-Y_{0}\right)-Y^{\prime},
\end{gathered}
$$

Now introducing the vector of normalized variables $X=\left(\tilde{\rho_{a}}, \tilde{\rho_{b}}, \tilde{u}, \tilde{v}, \tilde{T}\right)^{T}$

where

$$
\tilde{u}=\frac{u^{\prime}}{c_{0}} ; \quad \tilde{v}=\frac{v^{\prime}}{c_{0}} ; \quad \tilde{T}=\frac{T^{\prime}}{T_{0} \sqrt{\gamma(\gamma-1)}} ; \quad \tilde{\rho}_{a}=\frac{\rho_{a}^{\prime}}{\rho_{0} \sqrt{\frac{\gamma Y_{0}}{1+A_{t}^{r}\left(1-Y_{0}\right)}}} ; \quad \tilde{\rho}_{b}=\frac{\rho_{b}^{\prime}}{\rho_{0} \sqrt{\frac{\gamma\left(1-Y_{0}\right)}{1-A_{t}^{r} Y_{0}}}}
$$

and considering propagating plane wave disturbances, the linearized problem (4.1) can be rewritten in the following compact form

$$
\frac{d X}{d t}=M X
$$

where the linearized operator matrix is given by 


$$
M=\left(\begin{array}{ccccc}
-\mathrm{i} k_{x} u_{0} & 0 & -\frac{\mathrm{i} k_{x} c_{0}}{\sqrt{\gamma}} K_{1} & -\frac{\mathrm{i} k_{y} c_{0}}{\sqrt{\gamma}} K_{1} & 0 \\
0 & -\mathrm{i} k_{x} u_{0} & -\frac{\mathrm{i} k_{x} c_{0}}{\sqrt{\gamma}} K_{2} & -\frac{\mathrm{i} k_{y} c_{0}}{\sqrt{\gamma}} K_{2} & 0 \\
-\frac{\mathrm{i} k_{x} c_{0}}{\sqrt{\gamma}} K_{1} & -\frac{\mathrm{i} k_{x} c_{0}}{\sqrt{\gamma}} K_{2} & -\mathrm{i} k_{x} u_{0} & 0 & -\frac{\mathrm{i} k_{x} c_{0} \sqrt{\gamma-1}}{\sqrt{\gamma}} \\
-\frac{\mathrm{i} k_{y} c_{0}}{\sqrt{\gamma}} K_{1} & -\frac{\mathrm{i} k_{y} c_{0}}{\sqrt{\gamma}} K_{2} & 0 & -\mathrm{i} k_{x} u_{0} & -\frac{\mathrm{i} k_{y} c_{0} \sqrt{\gamma-1}}{\sqrt{\gamma}} \\
0 & 0 & -\frac{\mathrm{i} k_{x} c_{0} \sqrt{\gamma-1}}{\sqrt{\gamma}} & -\frac{\mathrm{i} k_{y} c_{0} \sqrt{\gamma-1}}{\sqrt{\gamma}} & -\mathrm{i} k_{x} u_{0}
\end{array}\right)
$$

where the two positive parameters $K_{1}$ and $K_{2}$ are defined as

$$
K_{1}=\sqrt{Y_{0}\left[1+A_{t}^{r}\left(1-Y_{0}\right)\right]}, \quad K_{2}=\sqrt{\left(1-Y_{0}\right)\left(1-A_{t}^{r} Y_{0}\right)},
$$

The five eigenvalues are

$$
-\mathrm{i} k_{x} u_{0}, \quad-\mathrm{i} k_{x} u_{0}, \quad-\mathrm{i} k_{x} u_{0}, \quad-\mathrm{i}\left(k_{x} u_{0} \mp k c_{0}\right),
$$

which correspond to the normalized propagation speeds of (from the left to the right) the entropy mode, the vorticity mode, the concentration mode and the fast and slow acoustic modes. The associated set of orthogonal eigenvectors is

$$
\begin{gathered}
X_{s}=\left(\begin{array}{c}
\sqrt{\frac{\gamma-1}{\gamma+K_{1}^{2}-1}} \\
0 \\
0 \\
0 \\
\frac{-K_{1}}{\sqrt{\gamma+K_{1}^{2}-1}}
\end{array}\right), X_{v}=\left(\begin{array}{c}
0 \\
0 \\
\frac{k_{y}}{k} \\
\frac{-k_{x}}{k} \\
0
\end{array}\right), X_{a^{ \pm}}=\left(\begin{array}{c}
\frac{K_{1}}{\sqrt{2 \gamma}} \\
\frac{K_{2}}{\sqrt{2 \gamma}} \\
\frac{\underline{k}_{x}}{k \sqrt{2}} \\
\frac{ \pm k_{y}}{k \sqrt{2}} \\
\sqrt{\frac{\gamma-1}{2 \gamma}}
\end{array}\right), \\
X_{Y}=\left(\begin{array}{c}
\frac{-K_{1} K_{2}}{\sqrt{\left(\gamma+K_{1}^{2}-1\right) \gamma}} \\
\sqrt{\frac{\gamma+K_{1}^{2}-1}{\gamma}} \\
0 \\
0 \\
-K_{2} \sqrt{\frac{\gamma-1}{\left(\gamma+K_{1}^{2}-1\right) \gamma}}
\end{array}\right) .
\end{gathered}
$$

All possible solutions of the linearized problem can be expressed as a linear combination of the eigenvectors: $X(t)=\sum_{i=s, v, a^{ \pm}, Y} C_{i}(t) X_{i}$. Therefore a local definition of the total energy $E(t)$ of the disturbance is given by the square of $L_{2}$ norm of $X(t)$. Thanks to the orthogonality property, one has $\|X(t)\|^{2}=X(t) \cdot X(t)=\sum_{i=s, v, a^{ \pm}, Y} C_{i}^{2}(t)\left\|X_{i}\right\|^{2}$, which appears as the sum of the energy of each mode. The associated energy in a volume $V$ is obtained in a straightforward way as:

$$
E_{t o t}(t)=\frac{\gamma p_{0}}{2} \int_{V}\left(\frac{K_{1}^{2} \rho_{a}^{\prime 2}}{\gamma \rho_{0}^{2} Y_{0}^{2}}+\frac{K_{2}^{2} \rho_{b}^{\prime 2}}{\gamma \rho_{0}^{2}\left(1-Y_{0}\right)^{2}}+\frac{u_{i}^{\prime} u_{i}^{\prime}}{c_{0}^{2}}+\frac{T^{\prime 2}}{\gamma(\gamma-1) T_{0}^{2}}\right) d V
$$

which can be rewritten as a function of $u_{i}^{\prime}, p^{\prime}, s^{\prime}$ and $Y^{\prime}$ as follows: 


$$
\begin{aligned}
E_{t o t}(t)= & \frac{\gamma p_{0}}{2} \int_{V}\left\{M_{0}^{2}\left(\frac{u_{i}^{\prime}}{u_{0}}\right)^{2}+\left(\frac{p^{\prime}}{\gamma p_{0}}\right)^{2}\right. \\
& +\frac{1}{\gamma}\left[\frac{K_{1}^{2}}{Y_{0}^{2}}+\frac{K_{2}^{2}}{\left(1-Y_{0}\right)^{2}}+\frac{\left(A_{t}^{r}\right)^{2}}{\gamma-1}\right]\left(Y^{\prime}\right)^{2} \\
& \left.+\frac{1}{\gamma-1}\left(\frac{s^{\prime}}{C_{p}}\right)^{2}\right\} d V .
\end{aligned}
$$

The original formula given by Chu for single-species fluids is recovered taking $Y_{0}=1$ (which leads to $K_{1}=1, K_{2}=0$ ) along with $Y^{\prime}=0$.

\section{A general formulation of the normal-mode-based LIA}

The shock jump relations for a normal planar shock wave with possible heat release/absorption and change in specific heats across the shock read

$$
\begin{aligned}
\bar{\rho}_{1}\left(u_{x 1}^{\prime}-\frac{\partial x_{s}}{\partial t}\right)+\bar{u}_{1} \rho_{1}^{\prime} & =\bar{\rho}_{2}\left(u_{x 2}^{\prime}-\frac{\partial x_{s}}{\partial t}\right)+\bar{u}_{2} \rho_{2}^{\prime}, \\
p_{1}^{\prime}+\rho_{1}^{\prime} \bar{u}_{1}^{2}+2 \bar{\rho}_{1} \bar{u}_{1} u_{x 1}^{\prime} & =p_{2}^{\prime}+\rho_{2}^{\prime} \bar{u}_{2}^{2}+2 \bar{\rho}_{2} \bar{u}_{2} u_{x 2}^{\prime} \\
h_{1}^{\prime}+\bar{u}_{1}\left(u_{x 1}^{\prime}-\frac{\partial x_{s}}{\partial t}\right) & =h_{2}^{\prime}+\bar{u}_{2}\left(u_{x 2}^{\prime}-\frac{\partial x_{s}}{\partial t}\right), \\
\bar{u}_{1} \frac{\partial x_{s}}{\partial y}+u_{r 1}^{\prime} & =\bar{u}_{2} \frac{\partial x_{s}}{\partial y}+u_{r 2}^{\prime} \\
u_{\phi 1}^{\prime} & =u_{\phi 2}^{\prime} \\
Y_{1}^{\prime} & =Y_{2}^{\prime} .
\end{aligned}
$$

As in Eq. (3.4), all prime quantities (e.g. $p_{1}^{\prime}$ ) correspond to the fluctuations around the average base flow (e.g. $\left.\bar{p}_{1}\right)$, and

$$
\left.x_{s}=x_{s}(y, t)=A_{x} e^{\mathrm{i}(k \sin \beta y-\Omega t}\right),
$$

denotes the shock displacement with respect to its equilibrium position, as depicted in Fig. 2. $A_{x}$ is the perturbation amplitude. 
The jump relations (5.1) can be normalized as

$$
\begin{gathered}
-\frac{\tau_{2}^{\prime}}{\bar{\tau}_{2}}+\frac{u_{x 2}^{\prime}}{\bar{u}_{2}}-\mathrm{i} \cos \beta(1-m) A_{x}=-\frac{\tau_{1}^{\prime}}{\bar{\tau}_{1}}+\frac{u_{x 1}^{\prime}}{\bar{u}_{1}}, \\
-\frac{\tau_{2}^{\prime}}{\bar{\tau}_{2}}+2 \frac{u_{x 2}^{\prime}}{\bar{u}_{2}}+\frac{1}{M_{2}^{2}} \frac{p_{2}^{\prime}}{\gamma \bar{p}_{2}}=m\left(-\frac{\tau_{1}^{\prime}}{\bar{\tau}_{1}}+2 \frac{u_{x 1}^{\prime}}{\bar{u}_{1}}+\frac{1}{M_{1}^{2}} \frac{p_{1}^{\prime}}{\gamma \bar{p}_{1}}\right), \\
\frac{u_{r 2}^{\prime}}{\bar{u}_{2}}+\mathrm{i} \sin \beta(1-m) A_{x}=m \frac{u_{r 1}^{\prime}}{\bar{u}_{1}} \\
\frac{u_{x 2}^{\prime}}{\bar{u}_{2}}+\left(\frac{1}{M_{2}^{2}}+\frac{1}{(\gamma-1) M_{2}^{2}}\right) \frac{p_{2}^{\prime}}{\gamma \bar{p}_{2}}+\frac{1}{(\gamma-1) M_{2}^{2}} \frac{\tau_{2}^{\prime}}{\bar{\tau}_{2}}+\mathrm{i} \cos \beta(1-m) m A_{x} \\
=m^{2}\left(\frac{u_{x 1}^{\prime}}{\bar{u}_{1}}+\left(\frac{1}{M_{1}^{2}}+\frac{1}{(\gamma-1) M_{1}^{2}}\right) \frac{p_{1}^{\prime}}{\gamma \bar{p}_{1}}+\frac{1}{(\gamma-1) M_{1}^{2}} \frac{\tau_{1}^{\prime}}{\bar{\tau}_{1}}\right), \\
+\frac{1}{\gamma(\gamma-1)}\left(\frac{m^{2}}{M_{1}^{2}}-\frac{1}{M_{2}^{2}}\right)\left(A_{t}^{C_{v}}-A_{t}^{r}\right) \\
\frac{u_{\phi 2}^{\prime}}{\bar{u}_{2}}=m \frac{u_{\phi 1}^{\prime}}{\bar{u}_{1}} \\
Y_{1}^{\prime}=Y_{2}^{\prime} .
\end{gathered}
$$

From the normalized shock relations, the transfer functions introduced in (3.5) can be expressed through the linear system

$$
M Z_{i}=B_{i}
$$

where the transfer function vector $Z_{i}$ contains the intensity of each emitted Kovasznay mode for a given incident mode $i=Y, t, v, s, a, x$

$$
Z_{i}=\left(Z_{i Y}, Z_{i t}, Z_{i v}, Z_{i s}, Z_{i a}, Z_{i x}\right)^{T}
$$

The matrix $M$ reads

$$
M=\left(\begin{array}{cccccc}
1 & 0 & 0 & 0 & 0 & 0 \\
0 & 1 & 0 & 0 & 0 & 0 \\
0 & 0 & \sin \alpha_{s} & -1 & 1+\frac{\cos \alpha_{a}+\mathrm{i} \eta}{M_{2} \zeta} & \mathrm{i}(m-1) \cos \beta \\
0 & 0 & 2 \sin \alpha_{s} & -1 & \frac{M_{2}^{2}+1}{M_{2}^{2}}+2 \frac{\cos \alpha_{a}+\mathrm{i} \eta}{M_{2} \zeta} & 0 \\
0 & 0 & -\cos \alpha_{s} & 0 & \frac{\sin \alpha_{a}}{M_{2} \zeta} & \mathrm{i}(1-m) \sin \beta \\
0 & 0 & \sin \alpha_{s} & \frac{1}{(\gamma-1) M_{2}^{2}} & \frac{1}{M_{2}^{2}}+\frac{\cos \alpha_{a}+\mathrm{i} \eta}{M_{2} \zeta} & \mathrm{i} m(1-m) \cos \beta
\end{array}\right)
$$


and the right-hand term, dependent on the incident wave's nature:

$$
\begin{aligned}
& B_{s}=\left(\begin{array}{c}
0 \\
0 \\
-1 \\
-m \\
0 \\
\frac{m^{2}}{(\gamma-1) M_{1}^{2}}
\end{array}\right), \quad B_{v}=\left(\begin{array}{c}
0 \\
0 \\
\sin \alpha \\
2 m \sin \alpha \\
-m \cos \alpha \\
m^{2} \sin \alpha
\end{array}\right), \quad B_{a}=\left(\begin{array}{c}
0 \\
0 \\
1+\frac{\cos \alpha}{M_{1}} \\
m\left(\frac{M_{1}^{2}+1}{M_{1}^{2}}+\frac{2 \cos \alpha}{M_{1}}\right) \\
m \frac{\sin \alpha}{M_{1}} \\
m^{2}\left(\frac{1}{M_{1}^{2}}+\frac{\cos \alpha}{M_{1}}\right)
\end{array}\right), \\
& B_{t}=\left(\begin{array}{c}
0 \\
m \\
0 \\
0 \\
0 \\
0
\end{array}\right), \quad B_{Y}=\left(\begin{array}{c}
1 \\
0 \\
0 \\
(1-m) A_{t}^{r} \\
0 \\
\left(\frac{m^{2}}{M_{1}^{2}}-\frac{1}{M_{2}^{2}}\right)\left(\begin{array}{c}
1 \\
\gamma(\gamma-1)
\end{array} A_{t}^{C_{v}}+\frac{1}{\gamma} A_{t}^{r}\right)
\end{array}\right) .
\end{aligned}
$$

From the above system, the transfer function vector can be deduced as

$$
\left(Z_{i Y}, Z_{i t}, Z_{i v}, Z_{i s}, Z_{i a}, Z_{i x}\right)^{T}=M^{-1} B_{i}
$$

where the inverse matrix is a block diagonal matrix of the same form as $M$. It can then be inferred that the toroidal mode is fully decoupled from the others

$$
\left\{\begin{array}{l}
Z_{t j}=B_{t}, \\
Z_{i t}=0
\end{array} \text { for } i \neq t .\right.
$$

A similar behavior is obtained for the concentration mode $Y$, when $\left(A_{t}^{r}, A_{t}^{C_{v}}\right)=(0,0)$ and $B_{Y}$ comprises of a single non-zero component. For arbitrary values of $\left(A_{t}^{r}, A_{t}^{C_{v}}\right)$, however,

$$
\left\{\begin{array}{l}
Z_{Y j} \neq B_{Y}, \\
Z_{i Y}=0
\end{array} \text { for } i \neq Y,\right.
$$

so that an upstream mass concentration perturbation can produce a combination of various modes downstream of the shock. Downstream, however, a mass fraction perturbation can only arise from an upstream mass fraction perturbation. These comments allow to consider a reduced number of $Z_{i j}$ terms in the following Figures.

The transfer functions obtained for acoustic, poloidal and entropy incident perturbations are plotted in Fig. 5 as functions of the incident angle $\alpha$. The associated emitted wave vectors are found in Fig. $4\left(\alpha_{a}\right.$ for $Z_{a i}$ and $\alpha_{s}$ for $Z_{v i}$ and $Z_{s i}$ ).

Incident mass fraction perturbations can vary in nature depending on the value of Atwood's numbers $\left(A_{t}^{r}, A_{t}^{C_{v}}\right)$ defined earlier (2.3). The associated transfer function $Z_{Y i}$ are therefore provided separately, in Fig. 6, with associated emitted wave angle $\alpha_{s}$ in Fig. 4. Note that $B_{Y}$ is linear in $A_{t}^{r}$ and $A_{t}^{C_{v}}$, so that providing solutions $Z_{Y i}$ for the two base vectors $\left(A_{t}^{r}, A_{t}^{C_{v}}\right)=(0,1)$ and $\left(A_{t}^{r}, A_{t}^{C_{v}}\right)=(1,0)$ suffice to describe the transfer function for any $\left(A_{t}^{r}, A_{t}^{C_{v}}\right)$. 

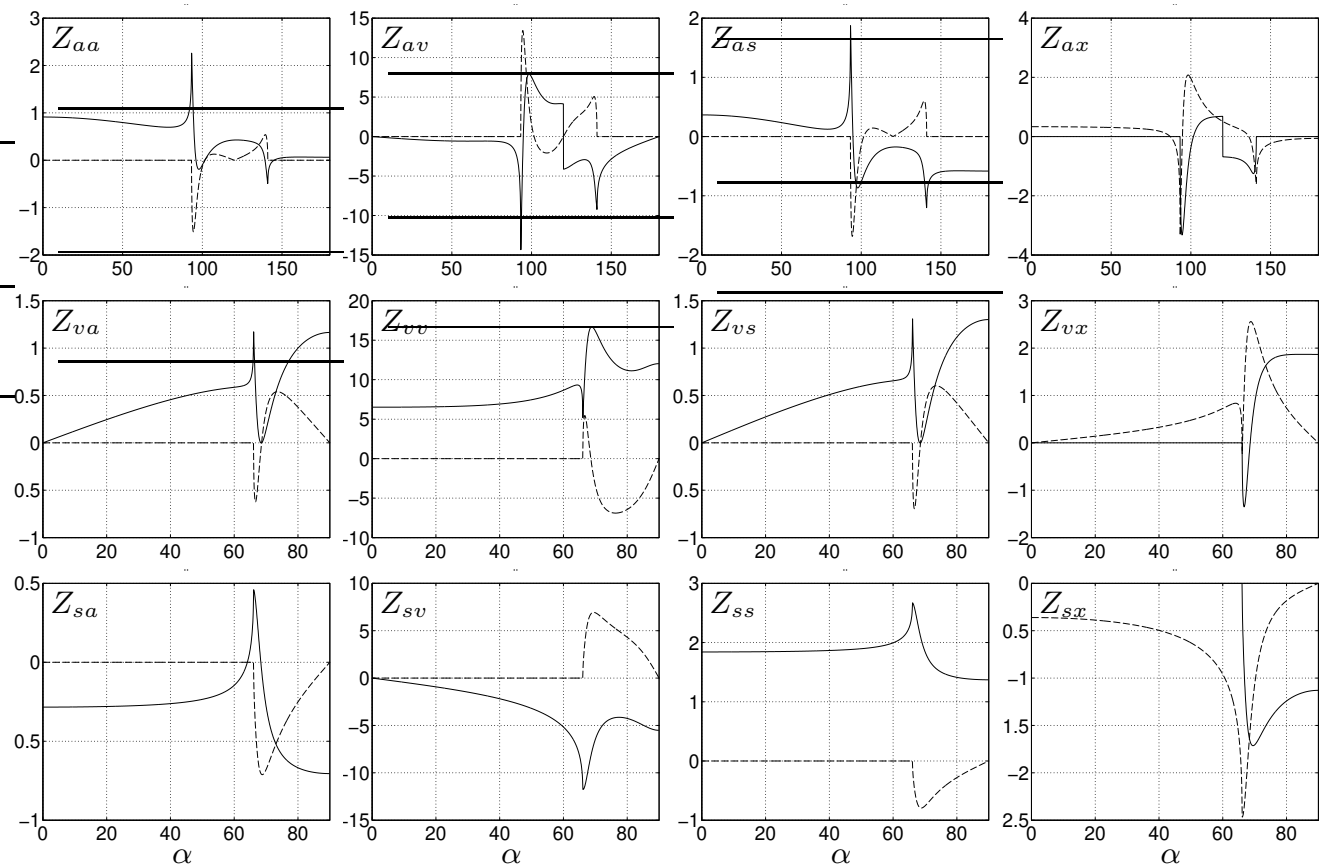

Figure 5: Real part (plain line) and imaginary part (dashed) of $Z_{i i}$ as a function of the incident wave angle $\alpha$, for $\gamma=1.4, M_{1}=2$ and $q=-2.25$. The corresponding emitted wave vectors angles $\alpha_{a}$ and $\alpha_{s}$ are those represented in Fig 4.
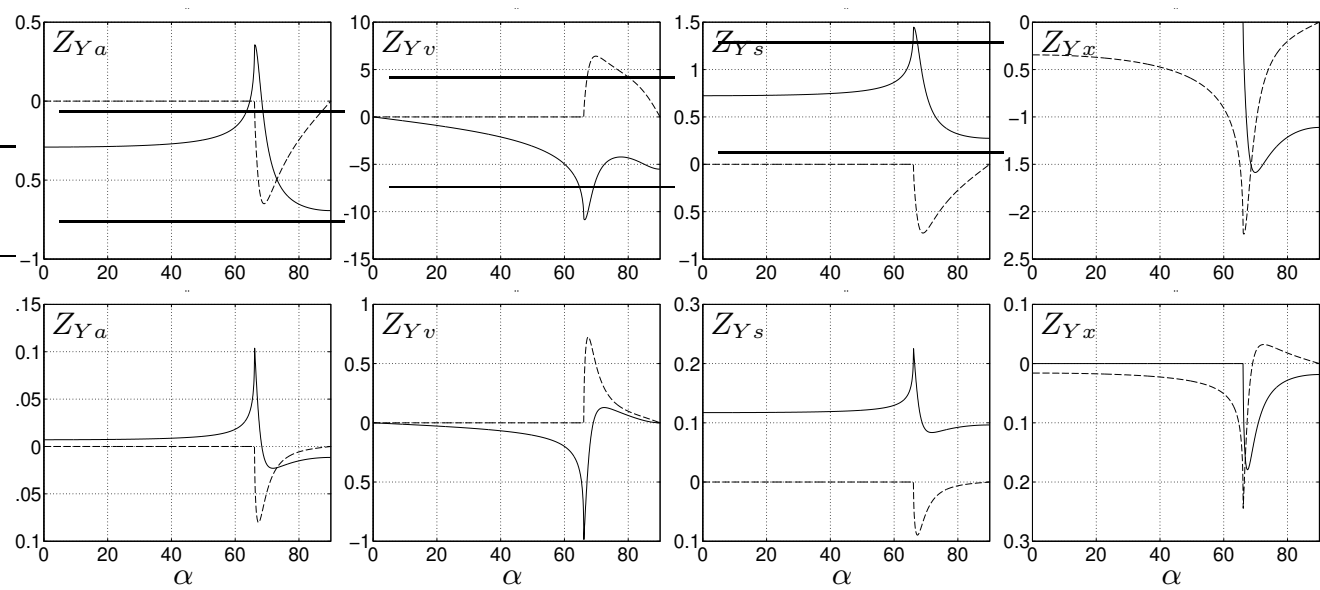

Figure 6: Real part (plain line) and imaginary part (dashed) of $Z_{Y i}$ as a function of the incident wave angle $\alpha$, for different incident mass fraction wave: $\left(A_{t}^{r}, A_{t}^{C_{v}}\right)=(1,0)$ (top), $(0,1)$ (bottom). The remaining parameters are identical to Fig. 5: $\gamma=1.4, M_{1}=2$ and $q=-2.25$. The corresponding emitted wave vector angle $\alpha_{s}$ can be found in Fig 4 .

\section{Interaction with Gaussian spots}

This section is dedicated to the interaction between 2D Gaussian spots advected at the uniform speed $U_{1}$ in the shock-normal direction and a planar shock wave. 

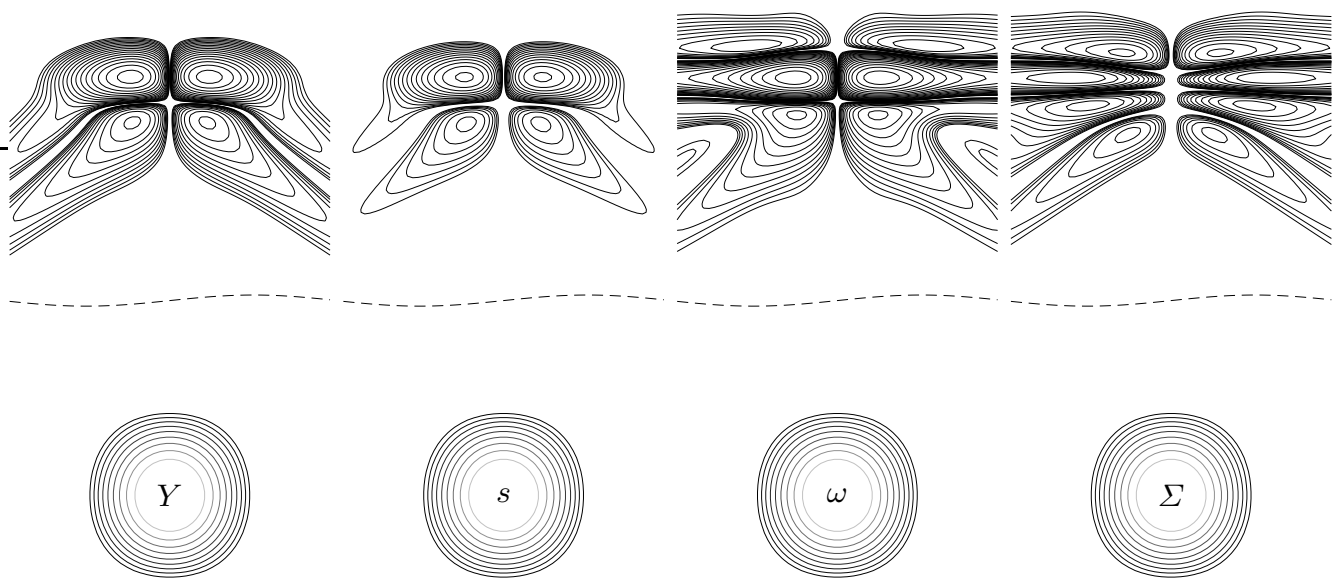

Figure 7: Emitted vorticity for incident gaussian density $Y$, entropy $s$, and vorticity $\omega$ spots. The fourth spot corresponds to the sum $\Sigma$ of the three gaussian spots, resulting in yet another vorticity pattern. The dashed line illustrates the corrugated shock.

The Gaussian spots are introduced as perturbations of the form

$$
G^{\prime}=\epsilon e^{-r^{2}}
$$

where $r$ is the radial coordinate relative to the centre of the spot, and the Gaussian perturbation $G^{\prime}$ is successively set as three elemental perturbations

$$
\begin{cases}G^{\prime}=\frac{\tau^{\prime}}{A_{t}^{r} \tau} & \text { for the density spot, } \\ G^{\prime}=\frac{s^{\prime}}{c_{p}} & \text { for the entropy spot, } \\ G^{\prime}=\frac{\omega^{\prime}}{U} & \text { for the vorticity spot. }\end{cases}
$$

For each perturbation, the emitted flow will systematically be studied through comparisons of acoustic, entropy and vorticity fields.

Note that, owing to the linear character of this study, it is straight-forward to combine these three elemental Gaussian perturbations into more complex ones, and obtain the emitted flow-field. This is illustrated in Fig. 7, which displays the vorticity field emitted from the combination of the three elemental spots presented hereafter.

In the following $\epsilon$, appears as a mere scaling and is therefore set to 1 . Typical results are shown for $A_{t}^{r}=2, A_{t}^{C_{v}}=1, M_{1}=2$ and $\gamma=1.4$. To illustrate the effect of the heat-release, results are plotted for adiabatic $(q=0)$, endothermic $(q=-2.25)$ and exothermic $(q=0.59)$. The numeric values for endothermic and exothermic shocks were chosen to be $q_{\min } / 2$ and $q_{\max } / 2$ at $M_{1}=2$.

\subsection{Gaussian density spot}

Let us now consider a density spot, e.g . $G^{\prime}=\frac{\tau^{\prime}}{A_{t}^{r} \tau}$ in (6.2), which can be considered as an idealized model for shock/dropplet interaction.

The choice of a positive $\epsilon$ in (6.2) corresponds to the definition of a heavy perturbation with respect to the upstream fluid, which can be interpreted as an ideal model for a droplet of heavy fluid. A negative value would correspond a pocket of light fluid. It is 

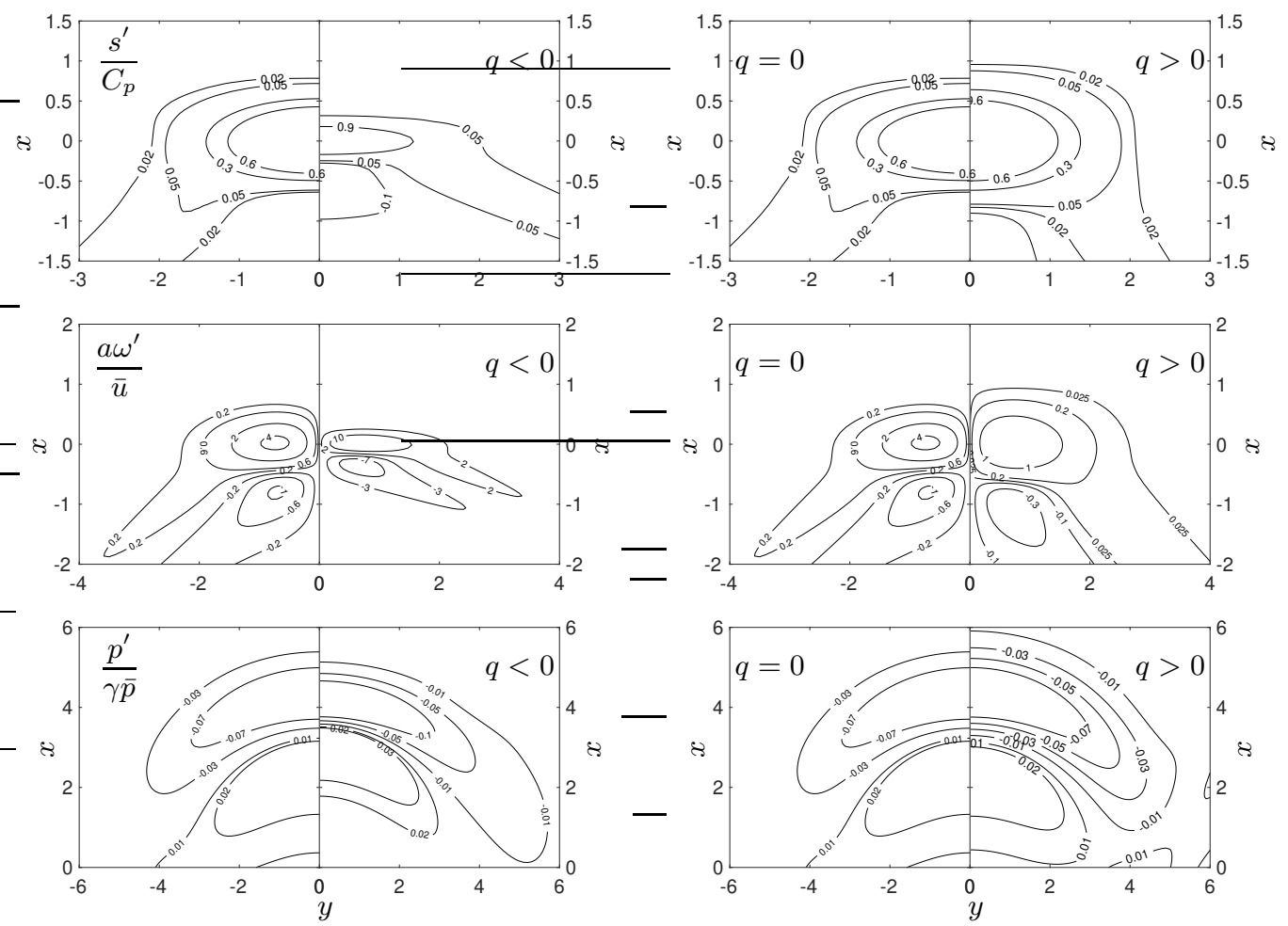

Figure 8: Incident Gaussian density spot: emitted entropy, vorticity and acoustic perturbations (from top to bottom). Left: adiabatic vs endothermic case. Right: adiabatic vs exothermic.

worth noting that pure density heterogeneities without acoustic perturbation, i.e. pure $\rho$ waves, are obtained considering concentration fluctuations. The solution is then computed analytically thanks to the formulas given in the Appendix.

The emitted fields of normalized entropy $\frac{s^{\prime}}{C_{p}}$ and vorticity $\frac{a \omega^{\prime}}{\bar{u}}$ are displayed in the first 4 plots of Figure. 8. Since the emitted patterns are advected at the constant speed $U_{2}$, they are plotted in the reference frame associated to the perturbation centre, in which they are frozen thanks to the fact that diffusive effects are not taken into account in the present inviscid model. The presented patterns are related to the far field solution, i.e. intermediary solutions that are found at times at which the incoming fluctuation spot has not totally crossed the shock are not presented for the sake of brevity (but can be computed).

It is seen that the topology of the emitted vorticity field is qualitatively the same in the three cases: a quadripolar pattern made of two counter-rotating vortex pairs is generated. This can be qualitatively interpreted as the result of a baroclinic effect of the form $-(\nabla p \times \nabla \rho) / \rho^{2}$, in which the positive pressure gradient is related to the pressure jump across the shock wave. From that expression, it is seen that the case of a light disturbance with a negative amplitude parameter $\epsilon$ would lead to a vorticity pattern with opposite sign, i.e. a pattern made of four vortices rotating in the opposite sense to those found for a heavy density spot.

The main effects of the heat source term being i) an amplification (resp. damping) of the amplitude of the emitted perturbations and ii) an increase (resp. decrease) of 

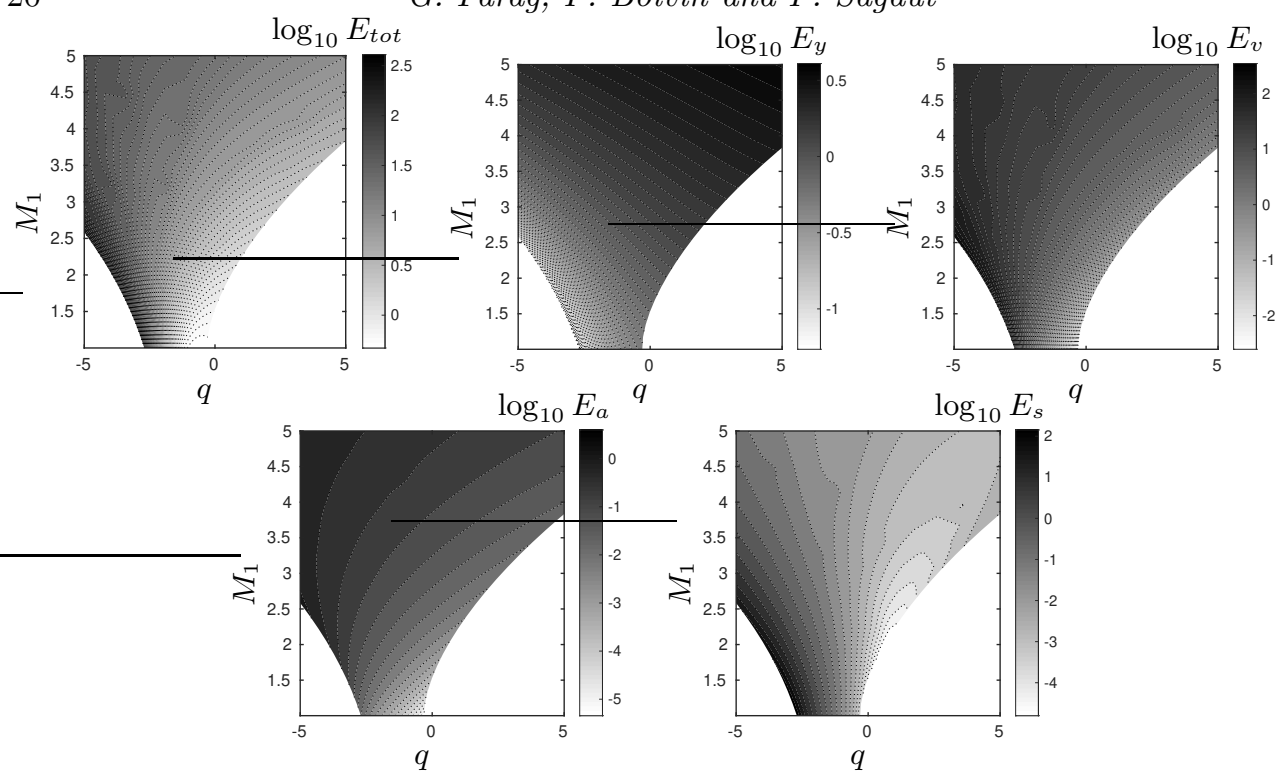

Figure 9: Energy of the emitted disturbances in the case of an incident Gaussian density spot in the $\left(M_{1}, q\right)$ plane. Total energy $E_{t o t}$ and the part associated to each Kovasznay mode are displayed, with $E_{y}$ : energy of the concentration mode; $E_{v}$ : energy of the vorticity mode; $E_{a}$ : energy of the acoustic mode; $E_{s}$ : energy of the entropy mode.

the anisotropy of the emitted pattern for endothermic (resp. exothermic) case, when compared to the adiabatic case. In the strong endothermic case the amplitude of the four vortices are nearly equal, while the second vortex pair is weaker in other cases. This is consistent with the fact that the effective shock-induced compressive effect is stronger in the endothermic case, as observed in Section 2.

The emitted acoustic field is illustrated here in the bottom plots of Fig. 8, in which the acoustic pressure field $\frac{p^{\prime}}{\gamma \bar{p}}$ is plotted at time $t=\frac{4 a}{c_{2}}$.

A more global view at the interaction physics is obtained looking at the energy of the emitted waves along with the part associated to each Kovasznay mode, according to the extended definition derived in Section 4. The area used to compute the sum in Eq.(4.13) is taken equal to $12 D \times 12 D$, which was checked to be large enough to get fully converged values, with $D$ defined as the radius of the incident Gaussian spot (see Appendix).

Results in the $\left(M_{1}, q\right)$ plane normalized by the energy of the incident density spot are displayed in Fig. 9 for the far-field solution, i.e. the transient contribution of acoustic non-propagative waves is omitted. Profiles along the $q=0$ and the $M_{1}=2$ lines are also shown in Fig. 10.

It is observed that the total emitted energy is an increasing function of the incoming Mach number $M_{1}$, and that the respective importance of each mode is strongly influenced by the heat source term $q$. In the neutral case $q=0$, the emitted energy is mainly due $E_{y}$ and $E_{v}$, i.e. to the concentration mode and the vorticity mode, the former being dominant for $M_{1}<4$. It is worth noting that the energy of all emitted modes is an increasing function of $M_{1}$, excepted $E_{s}$ which decreases for $1 \leqslant M_{1} \leqslant 2.6$ Varying $q$ at fixed $M_{1}$ makes a more complex behavior to appear. The emitted energy is mostly related to the vorticity mode in the endothermic case, the solution being dominated by the concentration mode for sligthly negative $q$ and exothermic cases. This is due to the case that the concentration mode is the only one which exhibit an increase for increasing 

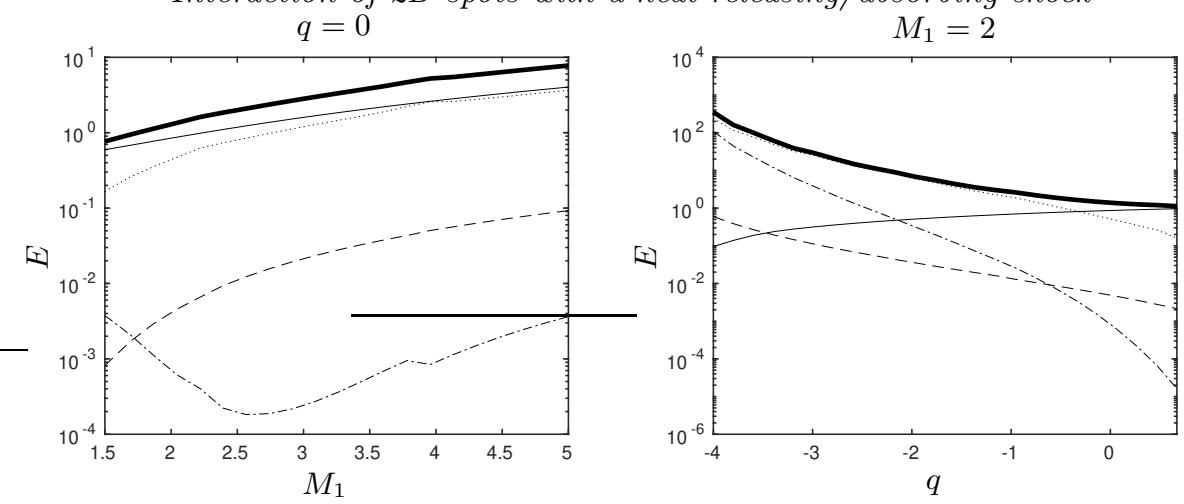

Figure 10: Energy of the emitted disturbances in the case of an incident Gaussian density spot versus $M_{1}$ for the adiabatic case $(q=0)$ and versus $q$ for $M_{1}=2$. Total energy and the part associated to each Kovasznay mode are displayed. $E_{t o t}$ (solid thick line), $E_{y}$ (solid), $E_{v}$ (dotted), $E_{a}$ (dashed), $E_{s}$ (dotted-dashed).

$q$, while a decrease of the total emitted energy $E_{t o t}$ associated to a monotonic decrease of all other modes is observed. A very fast decrease of $E_{s}$ is observed, leading to the fact that the entropy mode is very strong in the highly endothermic case, while it is the weakest mode in the neutral and and exothermic cases.

\subsection{Gaussian entropy spot}

This Section is dedicated to the interaction with a Gaussian entropy spot, and therefore is an extension of the previous analysis provided in Fabre et al. (2001) for the adiabatic case $q=0$. The upstream entropy spot is defined by setting $G^{\prime}=\frac{s^{\prime}}{c_{p}}$ in (6.2).

The emitted entropy far field, vorticity far field and acoustic pressure far field are displayed in Fig. 11. The emitted disturbance topology is the same as in the density case: a quadrupolar pattern made of two counter-rotating vortex pairs is generated downstream the shock, whose intensity and anisotropy are decreasing functions of the heat source term $q$. The key mechanisms for vorticity generation can again be interpreted as a kind of baroclinic production term associated to the pressure jump across the shock and the density gradient associated to the entropy disturbance, see Eq. (3.4).

The total energy of the emitted far-field solution (normalized by the energy of the incident spot) and the part associated to each Kovasznay component are plotted in Fig. 12 in the $\left(M_{1}, q\right)$ plane, while profiles along the $M_{1}=2$ and $q=0$ lines are shown in Fig. 13. It is worth noting that the concentration mode energy remains null downstream the shock, i.e. $E_{y}=0$, since it is null upstream the shock and the the concentration fluctuation is continuous at the shock according to Eq. (5.1).

Some interesting differences with the density spot case are observed, which are due to the fact that the entropy spot combines a density disturbance and a temperature disturbance. First, in the adiabatic case $q=0$, the normalized total emitted energy is not a monotonous function of the upstream Mach number $M_{1}$. A decrease is observed for $M_{1}<M_{\text {crit }} \simeq 2.7$, which is due to a decrease of the energy of the emitted entropy mode, which is a monotonic decaying function of $M_{1}$. The emitted acoustic and vorticity energy component, $E_{a}$ and $E_{v}$, are growing with $M_{1}, E_{a}$ being negligible in all cases. Therefore, the emitted field is dominated by the entropy mode for $M_{1}<M_{\text {crit }}$, while the vorticity mode is dominant at higher Mach number. This picture is very different from the one observed for the density spot, and it it stable with respect to a change in the parameter 

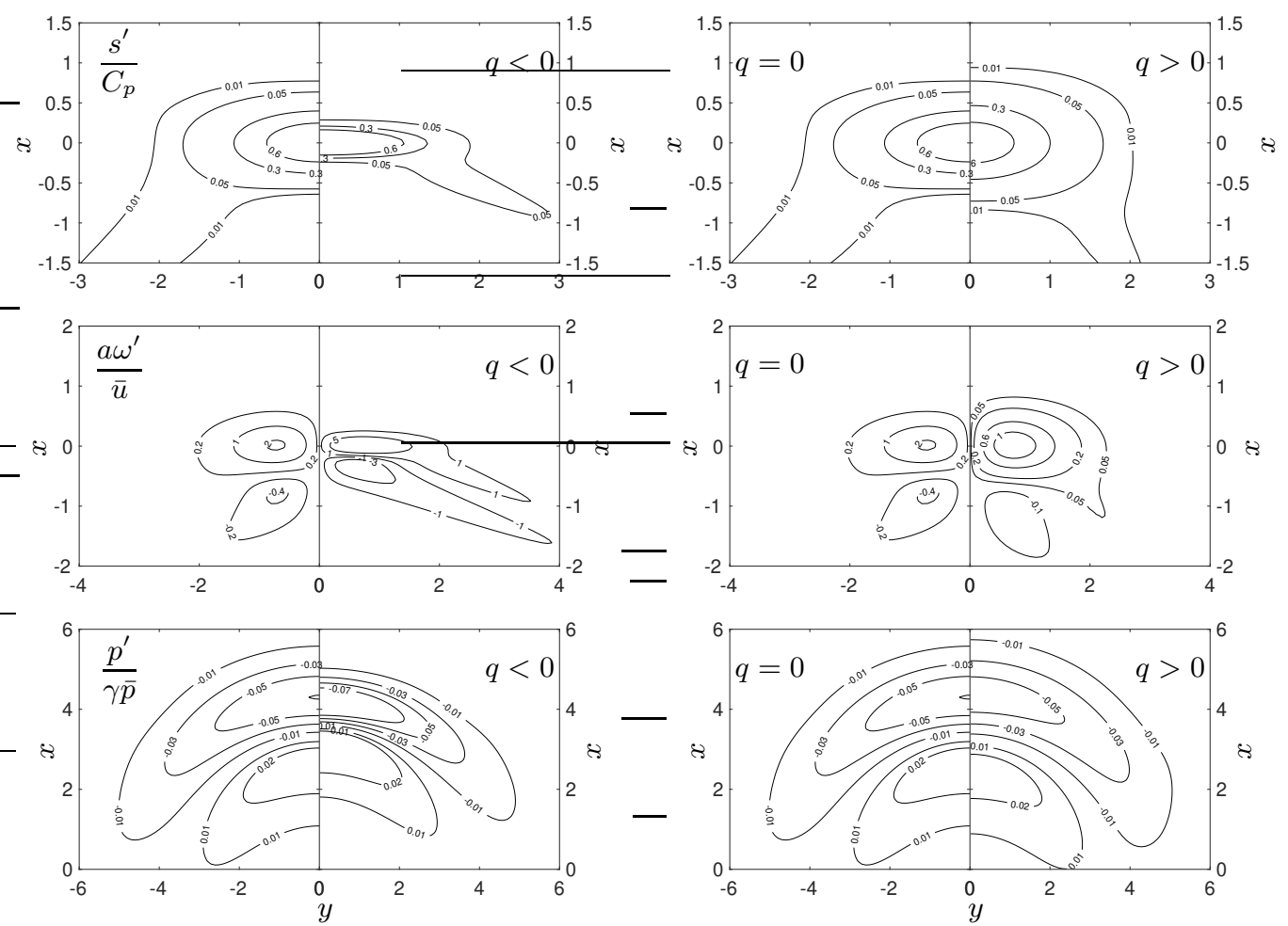

Figure 11: Incident Gaussian entropy spot: emitted entropy, vorticity and acoustic perturbations (from top to bottom). Left: adiabatic vs endothermic case. Right: adiabatic vs exothermic.

$q$. Here, the energy of all emitted modes decays when increasing $q$, including the emitted vortical energy which was an increasing function of $q$ in the density spot case.

\subsection{Gaussian vorticity spot}

The last case deals with the interaction between a planar shock wave and a Gaussian vorticity spot, which is a model of a weak vortex. The shock/vortex interaction has been addressed by several authors, mainly via Direct Numerical Simulation, but the present analysis is the first one to cover the full $\left(M_{1}, q\right)$ plane within the LIA framework.

Results for the emitted non-acoustic fields are shown in the first 4 plots of Fig. 14 . The concentration field remains uniform, as in the case of the entropy spot. A first observation is that the topology of the emitted field is different from the one observed for both incident density and entropy spot. As a matter of fact, while two vortex pairs with variable intensity were found previously, the present field is made of a strong counterrotating vortex pair, with two companion pairs of much weaker vortical structures.

The topology of the downstream acoustic field is investigated in the bottom plots of Fig. 14 which displays the generated pressure. A compression wave followed by a dilatation wave is observed, while in the two other cases the dilatation wave is emitted first.

The energy of the emitted field split into model components, normalized by the energy of the incident spot, is displayed in Figs. 15 and 16. It is observed that, in all cases, the emitted energy is dominated by the vortical component. In the adiabatic case, the acoustic energy remains larger than the entropy mode energy at all Mach number. The 

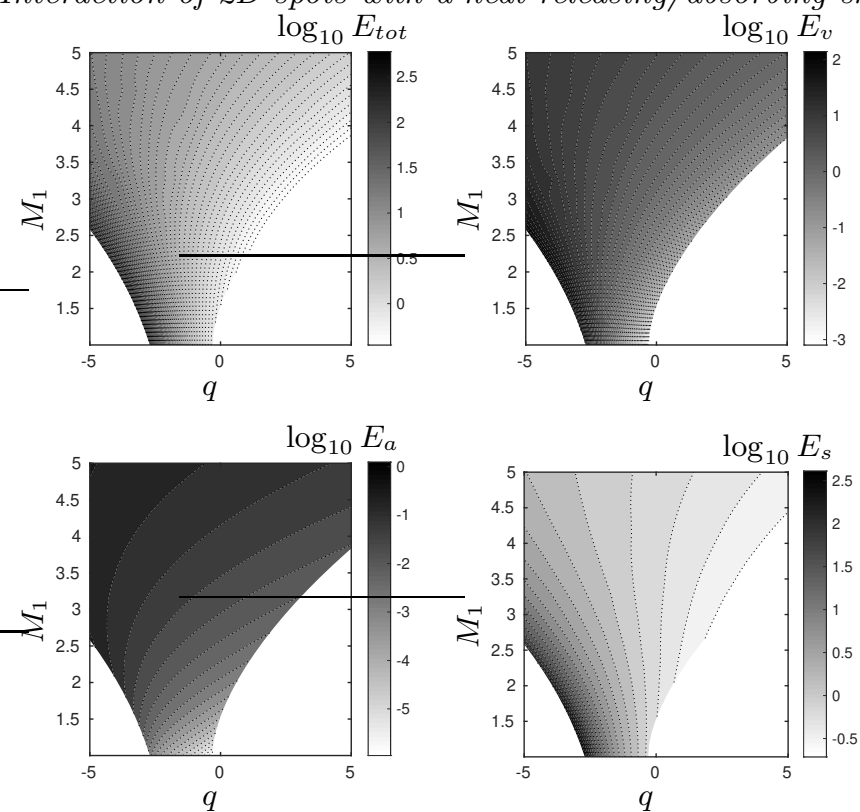

Figure 12: Energy of the emitted disturbances in the case of an incident Gaussian entropy spot in the $\left(M_{1}, q\right)$ plane. Total energy $E_{t o t}$ and the part associated to each Kovasznay mode are displayed, with $E_{v}$ : energy of the vorticity mode; $E_{a}$ : energy of the acoustic mode; $E_{s}$ : energy of the entropy mode.
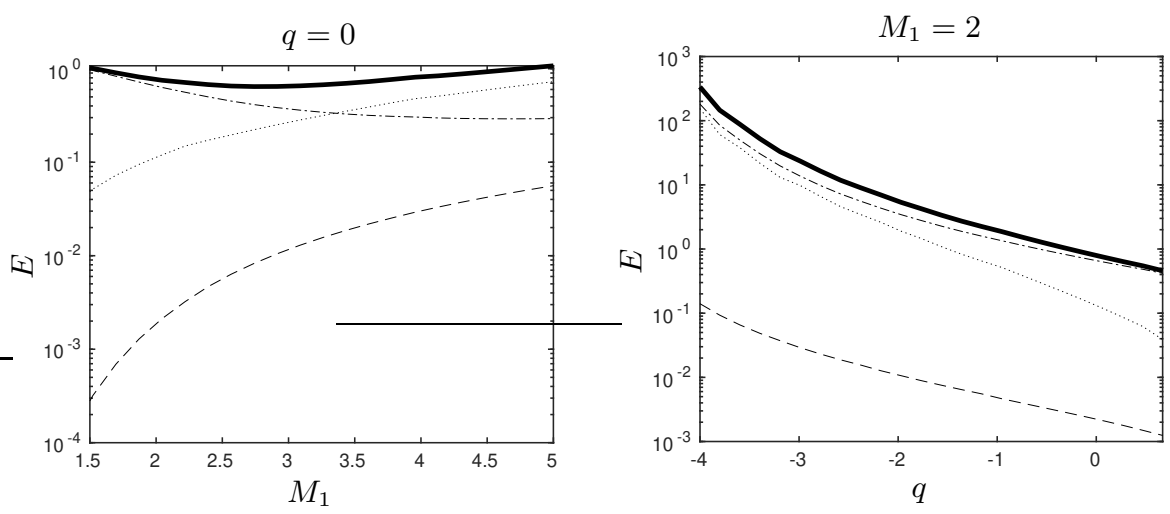

Figure 13: Energy of the emitted disturbances in the case of an incident Gaussian entropy spot versus $M_{1}$ for in the adiabatic case $(q=0)$ (top) and versus $q$ for $M_{1}=2$ (bottom). Total energy and the part associated to each Kovasznay mode are displayed. $E_{t o t}$ (solid thick line), $E_{v}$ (dotted), $E_{a}$ (dashed), $E_{s}$ (dotted-dashed).

opposite trend can be observed in strongly endothermic cases. All energy components are growing functions of $M_{1}$ and decreasing functions of $q$.

\subsection{Optimal mixed disturbances with minimal radiated noise}

The purpose of this section is to illustrate the possibility of finding upstream disturbances associated with peculiar emitted field. To this end, it is chosen to find the optimal combination of the three above elementary spots for minimal radiated noise.

Let us identify the emitted pressure perturbation as $p_{Y}^{\prime}, p_{s}^{\prime}$ and $p_{\omega}^{\prime}$ for the density, 

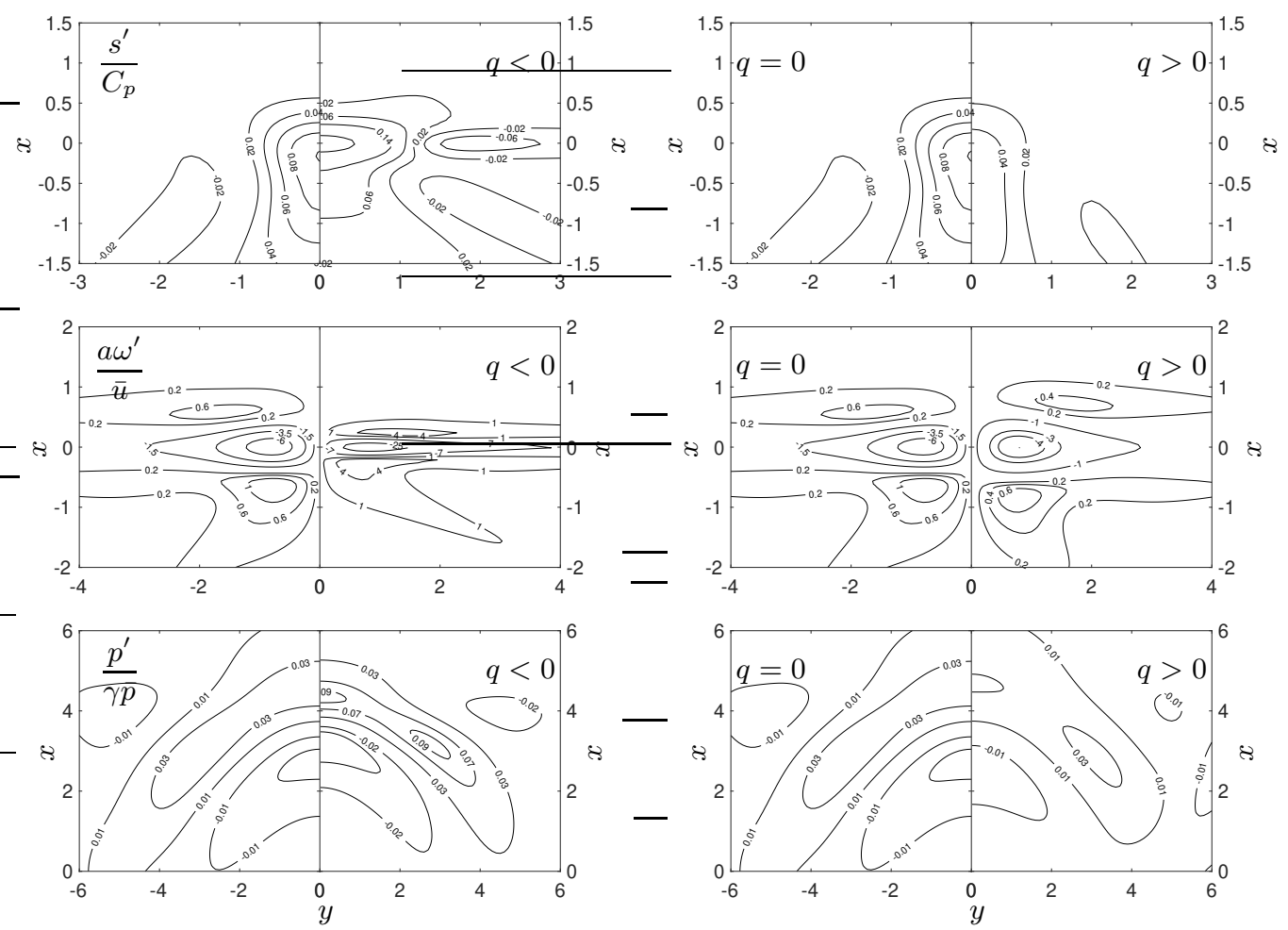

Figure 14: Incident weak vortex/Gaussian vorticity spot: emitted entropy, vorticity and acoustic perturbations (from top to bottom). Left: adiabatic vs endothermic case. Right: adiabatic vs exothermic.

entropy and vorticity gaussian elementary spots. Next, we introduce $\Pi$, the radiated noise emitted through the shock as

$$
\Pi\left(a_{s}, a_{Y}\right)=\iint_{V}\left(\frac{p_{\omega}^{\prime}+a_{s} p_{s}^{\prime}+a_{Y} p_{Y}^{\prime}}{\gamma \bar{p}}\right)^{2} d x d y,
$$

where $V$ is the volume of fluid after the shock. This corresponds to the acoustic perturbation obtained through combination of elementary spots as illustrated in Fig. 7, with coefficients $\left(a_{Y}, a_{s}, 1\right)$ for the three elementary spots. These combination can be interpreted as a family of low-density hot vortices.

Figure 17 presents the result of the minimization of $\Pi$ (6.3). The top two plots show the normalized relative amplitudes

$$
\alpha_{Y}=\frac{-a_{Y}}{1-a_{Y}+a_{s}}, \quad \alpha_{s}=\frac{a_{s}}{1-a_{Y}+a_{s}}, \quad \alpha_{\omega}=\frac{1}{1-a_{Y}+a_{s}},
$$

having found that $a_{Y}<0$ over the explored range of $\left(M_{1}, q\right)$. Note that the opposite sign found for $a_{s}$ and $a_{y}$ found to minimize $\Pi$ could have been intuited from Figs. 8 and 11 , the density and entropy spots leading to relatively similar emission patterns. The bottom two plots compare $\Pi$ for the optimal $\left(a_{s}, a_{y}\right)$ with $\Pi(0,0)$, the noise radiated by the elementary gaussian vorticity spot, showing that the vortex emitted noise was reduced by 80 to $90 \%$ by superimposing the adequate density and entropy perturbations.

Figure 18's top plot shows the resulting pressure field in the case $M_{1}=2$ and $q=0$, for which we found $a_{Y}=-0.976$ and $a_{s}=2.407$. It is obtained through linear combination 

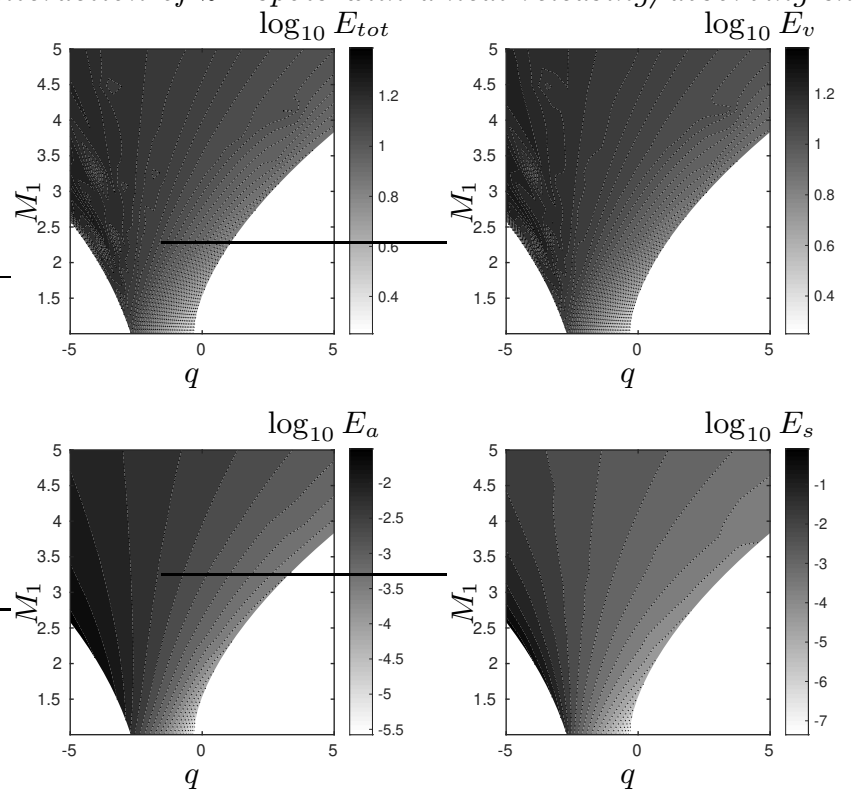

Figure 15: Energy of the emitted disturbances in the case of an incident weak vortex/Gaussian vorticity spot in the $\left(M_{1}, q\right)$ plane. Total energy $E_{t o t}$ and the part associated to each Kovasznay mode are displayed, with $E_{v}$ : energy of the vorticity mode; $E_{a}$ : energy of the acoustic mode; $E_{s}$ : energy of the entropy mode.
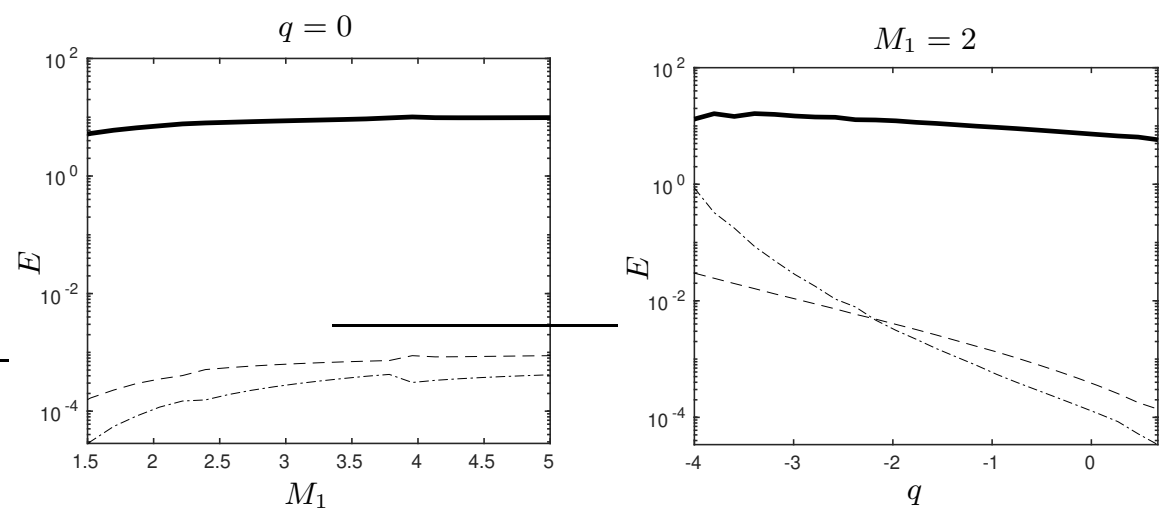

Figure 16: Energy of the emitted disturbances in the case of an weak vortex/Gaussian vorticity spot versus $M_{1}$ for in the adiabatic case and versus $q$ for $M_{1}=2$. Total energy and the part associated to each Kovasznay mode are displayed. $E_{t o t}$ (solid thick line), $E_{v}$ (dotted), $E_{a}$ (dashed), $E_{s}$ (dotted-dashed).

of the emitted pressure for the elementary spots of Figs 8, 11 and 14 with weights $\left(a_{Y}, a_{s}, 1\right)$. From the levels of the emitted pressure, it is clear that the radiated noise is significantly reduced compared to either elementary spot - by $82.6 \%$, as seen in Fig. 17 . The bottom plot of Fig. 18 shows the vorticity pattern downstream of the shock for the same perturbation, following Fig. 7.

Following the above procedure, it is straight-forward to minimize other fluctuations, such as vorticity, temperature, etc. 

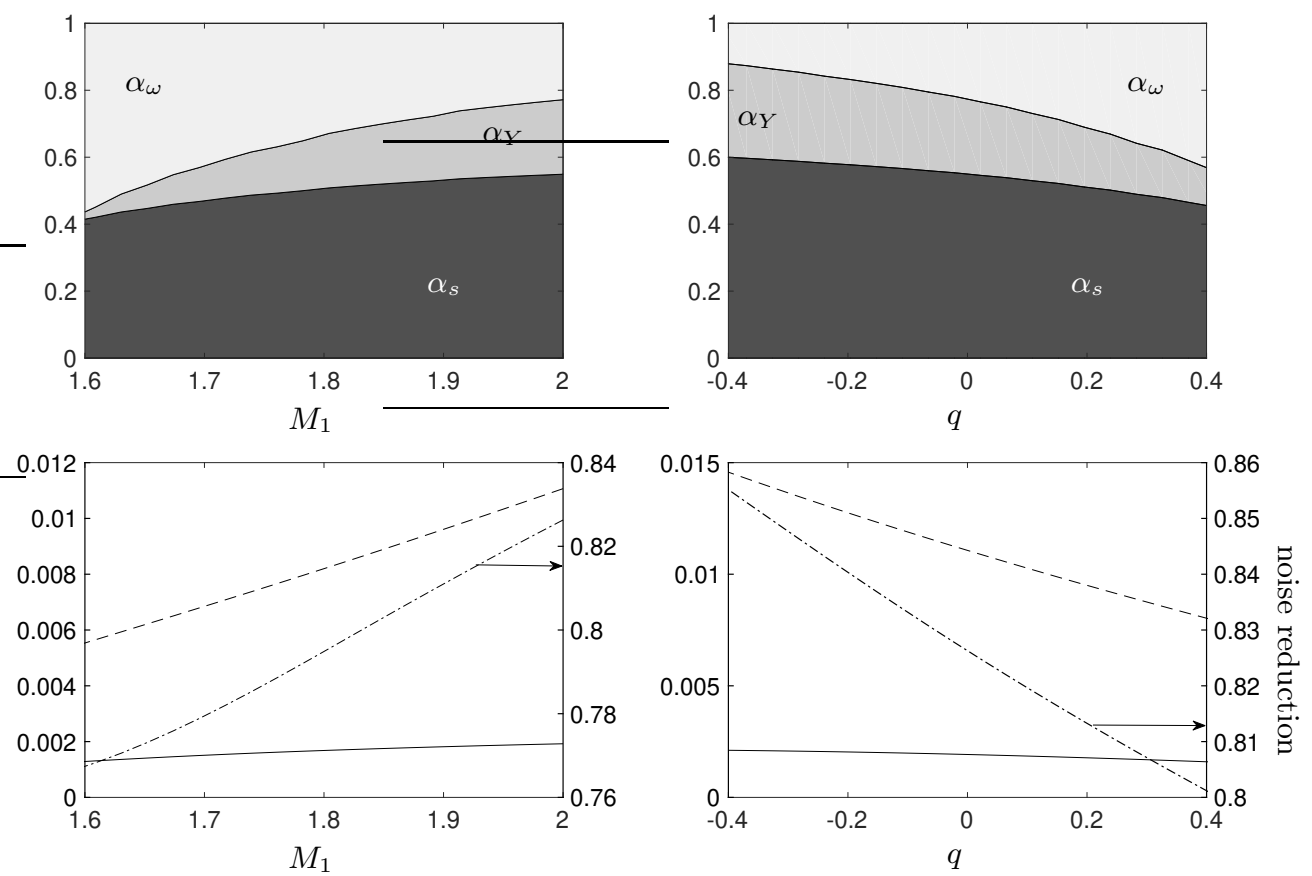

Figure 17: Relative spots amplitudes minimizing the radiated noise $\Pi$ (top) dependence with $M_{1}$ for $q=0$ (left), and with $q$ for $M_{1}=2$ (right). The corresponding integral radiated noise $\Pi(6.3)$ is shown for the elementary vortex $\Pi(0,0)$ (dashed) and the optimal combination $\Pi\left(a_{s}, a_{Y}\right)$ (solid) in the bottom two plots. The dot-dashed line, plotted in the right axes, shows the noise reduction $\frac{\Pi\left(a_{s}, a_{Y}\right)-\Pi(0,0)}{\Pi(0,0)}$.

\section{Concluding remarks}

A complete LIA framework for the interaction between a planar shock and a Gaussian disturbance including thermal effects at the shock front was proposed, along with adequate extension of the energy of the disturbances. General expressions for the emitted field are also provided, allowing for a straightforward reconstruction of the solution. Such a framework can provide a deep insight into shock/mixed disturbances interaction, but also very acurate benchmark solutions for numerical scheme validation. Another results is the extension of Chu's definition of disturbance energy to the present framework, leading to a mathematically-grounded meaningful definition of the energy of both upstream and downstream fields. It is worth noting that mixed solutions based on the combination of the three elementary solutions analyzed in the previous section can also be very easily obtained by linear combinations of the instantaneous elementary fields. This way, some solutions with peculiar features can be obtained. This is illustrated by the search of upstream vortex-like disturbances with minimal emitted pressure perturbations. In a similar way, combining an heavy density spot with a cold entropy spot one can obtain an emitted field with a very small residual vorticity. Solutions that minimize or maximize the energy of a given emitted Kovasznay mode can be obtained, the relative weight of each upstream mode being a function of the upstream Mach number and the heat source parameter $q$.

Strong of a wide variety of covered shock/spot interaction configurations, this work may serve as benchmark for the development of shock-capturing numerical methods. 

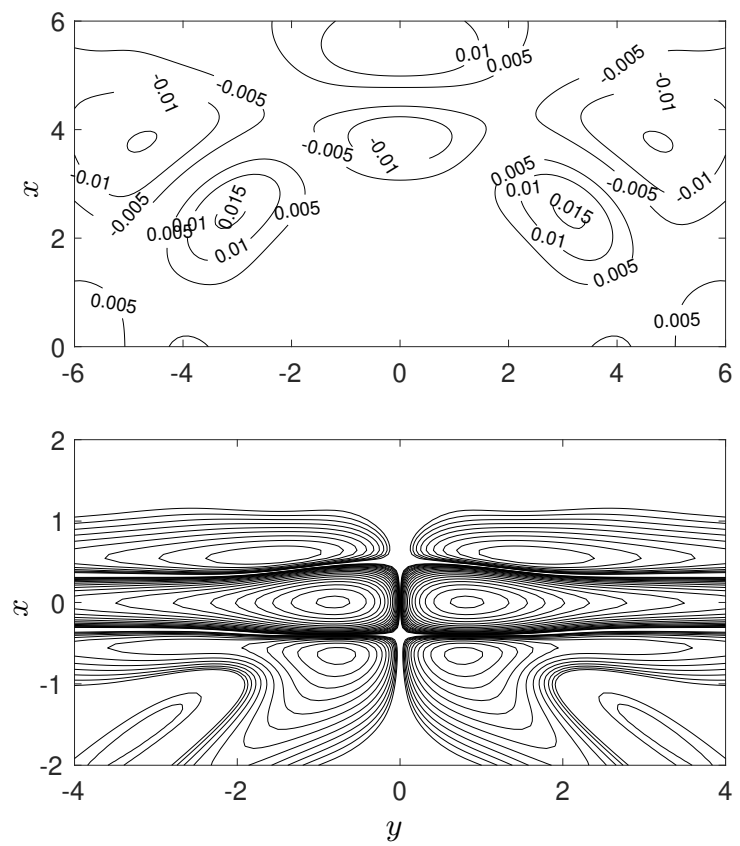

Figure 18: Emitted pressure (top) and vorticity (bottom) fields for the optimized vortex perturbation minimizing the radiated noise $\Pi$, in the case $M_{1}=2$ and $q=0$.

\section{Acknowledgements}

This work was supported by Labex MEC (ANR-10-LABX-0092) and the A*MIDEX project (ANR-11-IDEX-0001-02), funded by the "Investissements d'Avenir".

\section{Appendix: Mathematical formulation for Gaussian spot/shock interaction}

One addresses here the general formulation for the upstream and downstream field associated to the interaction between a 2D Gaussian non-acoustic disturbance and a planar shock within the Kovasznay decomposition framework. The upstream disturbances are advected at the constant speed $U$, and are then frozen in the reference frame moving with the base flow far from the shock wave.

Considering an upstream perturbation of Kovasznay mode $i$ of the form (in cylindrical coordinates $(r, \phi))$

$$
f_{i}(r)=\epsilon e^{-r^{2} / D^{2}}
$$

where $D$ denotes the radius (taken equal to 1 ) of the Gaussian profile and $f_{i}$ the fluctuation of the physical quantity associated to the Kovasznay mode (e.g. density or specific volume for a density spot, entropy for an entropy spot, vorticity for a vortex), its decomposition into Fourier modes obtained via a 2D polar Fourier transform is (see Fabre et al. (2001) for a full description of intermediary algebra, with $(k, \alpha)$ the polar coordinates in the Fourier space) 
where

$$
f_{i}(r)=\frac{\epsilon}{2 \pi} \int_{\alpha=-\frac{\pi}{2}}^{\frac{\pi}{2}} K(\hat{r}) d \alpha
$$

$$
\begin{gathered}
K(z)=\int_{k=0}^{\infty} \frac{k}{2} e^{-\left(\frac{k}{2}\right)^{2}} \cos (k z) \mathrm{d} k=1-\sqrt{\pi} z e^{-z^{2}} \operatorname{erfi}(z), \\
\hat{r}=r \cos (\alpha-\phi) .
\end{gathered}
$$

The complete emitted field is given by

$$
\begin{aligned}
& \frac{s_{2}^{\prime}}{C_{p 2}}=\frac{\epsilon}{\pi} \int_{\alpha=-\frac{\pi}{2}}^{\frac{\pi}{2}}\left[\operatorname{Re}\left(Z_{i s}\right) K\left(\frac{\widehat{r}^{s}}{c}\right)-\operatorname{Im}\left(Z_{i s}\right) L\left(\frac{\widehat{r}^{s}}{c}\right)\right] \mathrm{d} \alpha, \\
& \frac{\omega_{2}^{\prime}}{U_{2}}=-\frac{\epsilon}{\pi} \int_{\alpha=-\frac{\pi}{2}}^{\frac{\pi}{2}} \frac{1}{c}\left[\operatorname{Re}\left(Z_{i v}\right) K^{\prime}\left(\frac{\widehat{r}^{s}}{c}\right)-\operatorname{Im}\left(Z_{i v}\right) L^{\prime}\left(\frac{\widehat{r}^{s}}{c}\right)\right] \mathrm{d} \alpha, \\
& \frac{p_{2}^{\prime}}{\gamma P_{2}}=\frac{\epsilon}{2 \pi} \operatorname{Re}\left(\int_{\alpha=-\frac{\pi}{2}}^{\frac{\pi}{2}} \int_{k=0}^{\infty} Z_{i a} e^{\frac{-k^{2}}{4}} e^{-k_{a} \eta\left(x+M_{2} c_{2} t\right)} e^{\mathbf{i} \mathbf{k}_{a} \cdot \mathbf{x}} k \mathrm{~d} k \mathrm{~d} \alpha\right), \\
& \frac{T_{2}^{\prime}}{T_{2}}=\frac{\epsilon}{\pi} \int_{\alpha=-\frac{\pi}{2}}^{\frac{\pi}{2}}\left[\operatorname{Re}\left(Z_{i s}\right) K\left(\frac{\widehat{r}^{s}}{c}\right)-\operatorname{Im}\left(Z_{i s}\right) L\left(\frac{\widehat{r}^{s}}{c}\right)\right] \mathrm{d} \alpha+(\gamma-1) \frac{p_{2}^{\prime}}{\gamma P_{2}}, \\
& \frac{u_{x 2}^{\prime}}{U_{2}}=\frac{\epsilon}{\pi} \int_{\alpha=-\frac{\pi}{2}}^{\frac{\pi}{2}}\left[\operatorname{Re}\left(Z_{i v}\right) K\left(\frac{\widehat{r}^{s}}{c}\right)-\operatorname{Im}\left(Z_{i v}\right) L\left(\frac{\widehat{r}^{s}}{c}\right)\right] \sin \alpha_{s} \mathrm{~d} \alpha \\
& +\frac{\epsilon}{2 \pi} \operatorname{Re}\left(\int_{\alpha=-\frac{\pi}{2}}^{\frac{\pi}{2}} \int_{k=0}^{\infty} Z_{i a} \frac{\cos \alpha_{a}+\mathrm{i} \eta}{M_{2} \zeta} e^{\frac{-k^{2}}{4}} e^{-k_{a} \eta\left(x+M_{2} c_{2} t\right)} e^{\mathrm{i} \mathbf{k}_{a} \cdot \mathbf{x}} k \mathrm{~d} k \mathrm{~d} \alpha\right), \\
& \frac{u_{r 2}^{\prime}}{U_{2}}=-\frac{\epsilon}{\pi} \int_{\alpha=-\frac{\pi}{2}}^{\frac{\pi}{2}}\left[\operatorname{Re}\left(Z_{i v}\right) K\left(\frac{\widehat{r}^{s}}{c}\right)-\operatorname{Im}\left(Z_{i v}\right) L\left(\frac{\widehat{r}^{s}}{c}\right)\right] \cos \alpha_{s} \mathrm{~d} \alpha \\
& +\frac{\epsilon}{2 \pi} \operatorname{Re}\left(\int_{\alpha=-\frac{\pi}{2}}^{\frac{\pi}{2}} \int_{k=0}^{\infty} Z_{i a} \frac{\sin \alpha_{a}}{M_{2} \zeta} e^{\frac{-k^{2}}{4}} e^{-k_{a} \eta\left(x+M_{2} c_{2} t\right)} e^{\mathrm{i} \mathbf{k}_{a} \cdot \mathbf{x}} k \mathrm{~d} k \mathrm{~d} \alpha\right), \\
& \frac{u_{\phi 2}^{\prime}}{U_{2}}=\frac{\epsilon}{\pi} \int_{\alpha=-\frac{\pi}{2}}^{\frac{\pi}{2}}\left[\operatorname{Re}\left(Z_{i t}\right) K\left(\frac{\widehat{r}^{s}}{c}\right)-\operatorname{Im}\left(Z_{i t}\right) L\left(\frac{\widehat{r}^{s}}{c}\right)\right] \mathrm{d} \alpha,
\end{aligned}
$$

where it is recalled that $\mathbf{k}_{a} \cdot \mathbf{x}=k_{a}\left(\cos \alpha_{a}\left(x+U_{2} t\right)+\sin \alpha_{a}\left(y-U_{2} t \cot \alpha_{s}\right)\right)$. The auxiliary functions $L, K^{\prime}, L^{\prime}, \widehat{r}^{s}$ and $c$ are defined as :

$$
\begin{gathered}
L(z)=\int_{k=0}^{\infty} \frac{k}{2} e^{-\left(\frac{k}{2}\right)^{2}} \sin (k z) \mathrm{d} k=\sqrt{\pi} z e^{-z^{2}}, \\
K^{\prime}(z)=-2 z-\sqrt{\pi}\left(1-2 z^{2}\right) e^{-z^{2}} \operatorname{erfi}(z),
\end{gathered}
$$




$$
\begin{gathered}
L^{\prime}(z)=\sqrt{\pi}\left(1-2 z^{2}\right) e^{-z^{2}}, \\
c=\frac{\sin \alpha_{s}}{\sin \alpha}, \quad \widehat{r}^{s}=r \cos \left(\alpha_{s}-\phi\right) .
\end{gathered}
$$

The emitted density field $\rho_{2}^{\prime}$ and specific volume $\tau_{2}^{\prime}=1 / \rho_{2}^{\prime}$ are computed for each component from $T^{\prime}, Y^{\prime}$ and $p^{\prime}$ in a straightforward way using the linearized equation of state, i.e.

$$
\frac{p^{\prime}}{P}=\frac{\rho^{\prime}}{\rho}+\frac{T^{\prime}}{T}+A_{t}^{r} Y^{\prime} .
$$




\section{REFERENCES}

Abdikamalov, Ernazar, Huete, César, Nussupbekov, Ayan \& Berdibek, Shapagat 2018 Turbulence generation by shock-acoustic-wave interaction in core-collapse supernovae. Particles 1 (1), 97-110.

Chu, BoA-Ten 1965 On the energy transfer to small disturbances in fluid flow (part i). Acta Mechanica 1 (3), 215-234.

Chu, Boa-Teh \& Kovásznay, Leslie SG 1958 Non-linear interactions in a viscous heatconducting compressible gas. Journal of Fluid Mechanics 3 (5), 494-514.

Fabre, David, Jacquin, Laurent \& Sesterhenn, Jörn 2001 Linear interaction of a cylindrical entropy spot with a shock. Physics of Fluids 13 (8), 2403-2422.

George, K Joseph \& Sujith, RI 2011 On chu's disturbance energy. Journal of Sound and Vibration 330 (22), 5280-5291.

GrIFFond, J 2005 Linear interaction analysis applied to a mixture of two perfect gases. Physics of Fluids 17 (8), 086101.

GRIFFOND, J 2006 Linear interaction analysis for richtmyer-meshkov instability at low atwood numbers. Physics of Fluids 18 (5), 054106.

Griffond, JÉrôme \& Soulard, Olivier 2012 Evolution of axisymmetric weakly turbulent mixtures interacting with shock or rarefaction waves. Physics of Fluids 24 (11), 115108.

Griffond, J, Soulard, O \& Souffland, D 2010 A turbulent mixing reynolds stress model fitted to match linear interaction analysis predictions. Physica Scripta 2010 (T142), 014059 .

Huete, CÉsar \& Abdikamalov, Ernazar 2019 Response of nuclear-dissociating shocks to vorticity perturbations. Physica Scripta .

Huete, César, Abdikamalov, Ernazar \& Radice, David 2018 The impact of vorticity waves on the shock dynamics in core-collapse supernovae. Monthly Notices of the Royal Astronomical Society 475 (3), 3305-3323.

Huete, César, Sánchez, Antonio L \& Williams, Forman A 2013 Theory of interactions of thin strong detonations with turbulent gases. Physics of Fluids 25 (7), 076105.

Huete, César, Sánchez, Antonio L \& Williams, Forman A 2014 Linear theory for the interaction of small-scale turbulence with overdriven detonations. Physics of Fluids 26 (11), 116101.

Huete, C, Wouchuk, JG, Canaud, B \& Velikovich, AL 2012a Analytical linear theory for the shock and re-shock of isotropic density inhomogeneities. Journal of Fluid Mechanics 700, 214-245.

Huete, C, Wouchuk, JG \& Velikovich, AL 2012b Analytical linear theory for the interaction of a planar shock wave with a two-or three-dimensional random isotropic acoustic wave field. Physical Review E 85 (2), 026312.

Kovasznay, Leslie SG 1953 Turbulence in supersonic flow. Journal of the Aeronautical Sciences 20 (10), 657-674.

Lee, Sangsan, Lele, Sanjiva K \& Moin, Parviz 1993 Direct numerical simulation of isotropic turbulence interacting with a weak shock wave. Journal of Fluid Mechanics 251, 533-562.

Lee, Sangsan, Lele, Sanjiva K \& Moin, Parviz 1997 Interaction of isotropic turbulence with shock waves: effect of shock strength. Journal of Fluid Mechanics 340, 225-247.

DE LiRA, C Huete Ruiz 2010 Turbulence generation by a shock wave interacting with a random density inhomogeneity field. Physica Scripta 2010 (T142), 014022.

Mahesh, Krishnan, Lee, Sangsan, Lele, Sanjiva K \& Moin, Parviz 1995 The interaction of an isotropic field of acoustic waves with a shock wave. Journal of Fluid Mechanics 300, 383-407.

Mahesh, Krishnan, Lele, Sanjiva K \& Moin, Parviz 1997 The influence of entropy fluctuations on the interaction of turbulence with a shock wave. Journal of Fluid Mechanics 334, 353-379.

Moore, Franklin K 1953 Unsteady oblique interaction of a shock wave with a plane disturbance .

NARIta, Shinji 1973 The radiative energy loss from the shock front. Progress of Theoretical Physics 49 (6), 1911-1931.

Quadros, Russell, Sinha, Krishnendu \& Larsson, Johan 2016 Turbulent energy flux 
generated by shock/homogeneous-turbulence interaction. Journal of Fluid Mechanics 796, 113-157.

RIBNER, H.S. 1954a Shock-turbulence interaction and the generation of noise. NACA Technical Note 3255.

Ribner, Herbert S 1954b Convection of a pattern of vorticity through a shock wave .

Ribner, Herbert S 1959 The sound generated by interaction of a single vortex with a shock wave. Tech. Rep.. University of Toronto.

Ryu, Jaiyoung \& Livescu, Daniel 2014 Turbulence structure behind the shock in canonical shock-vortical turbulence interaction. Journal of Fluid Mechanics $\mathbf{7 5 6 .}$

Sagaut, P. \& Cambon, C. 2018 Homogeneous turbulence dynamics, 2nd edition. Springer.

SinHA, KRISHNENDU 2012 Evolution of enstrophy in shock/homogeneous turbulence interaction. Journal of Fluid Mechanics 707, 74-110.

Sinha, Krishnendu, Mahesh, Krishnan \& Candler, Graham V 2003 Modeling shock unsteadiness in shock/turbulence interaction. Physics of Fluids 15 (8), 2290-2297.

Soulard, Olivier, Griffond, Jérôme \& Souffland, Denis 2012 Pseudocompressible approximation and statistical turbulence modeling: Application to shock tube flows. Physical Review E 85 (2), 026307.

Williams, Forman Arthur 1985 Combustion Theory, second edition.. Cummings Publ. Co.

Wouchuk, JG, De Lira, C Huete Ruiz \& Velikovich, AL 2009 Analytical linear theory for the interaction of a planar shock wave with an isotropic turbulent vorticity field. Physical Review E 79 (6), 066315.

Zeldovich, Yakov Borissovich 1950 On the theory of the propagation of detonation in gaseous systems .

Zel'Dovich, YA B \& RAizer, Yu P 2012 Physics of shock waves and high-temperature hydrodynamic phenomena. Courier Corporation.

Zhao, Lianguu, Wang, Fei, Gao, Hong, Tang, Jingwen \& Yuan, Yuexiang 2008 Shock wave of vapor-liquid two-phase flow. Front. Energy Power Eng. China 2 (3), 344-347. 